\title{
Characterization of volatile compounds of Daucus crinitus Desf. Headspace Solid Phase Microextraction as alternative technique to Hydrodistillation
}

Mohammed El Amine Dib ${ }^{1}$, Nassim Djabou ${ }^{1,2}$, Jean-Marie Desjobert ${ }^{2}$, Houcine Allali ${ }^{1}$, Boufeldja Tabti ${ }^{1}$, Alain Muselli ${ }^{*}$, Jean Costa ${ }^{2}$

\begin{abstract}
Background: Traditionally, the essential oil of aromatic herbs is obtained using hydrodistillation (HD). Because the emitted volatile fraction plays a fundamental role in a plant's life, various novel techniques have been developed for its extraction from plants. Among these, headspace solid phase microextraction (HS-SPME) can be used to obtain a rapid fingerprint of a plant's headspace. Daucus crinitus Desf. is a wild plant that grows along the west coast of Algeria. Only a single study has dealt with the chemical composition of the aerial part oils of Algerian D. crinitus, in which isochavicol isobutyrate (39.0\%), octyl acetate (12.3\%), and $\beta$-caryophyllene (5.4\%) were identified. Using GC-RI and GC-MS analysis, the essential oils and the volatiles extracted from separated organs of D. crinitus Desf. were studied using HS-SPME.
\end{abstract}

Results: GC-RI and GC-MS analysis identified 72 and 79 components in oils extracted using HD and in the volatile fractions extracted using SPME, respectively. Two types of essential oils were produced by the plant: the root oils had aliphatic compounds as the main component (87.0\%-90.1\%), and the aerial part oils had phenylpropanoids as the main component (43.1\%-88.6\%). HS-SPME analysis showed a more precise distribution of compounds in the organs studied: oxygenated aliphatic compounds were well represented in the roots (44.3\%-84.0\%), hydrocarbon aliphatic compounds were in the leaves and stems (22.2\%-87.9\%), and phenylpropanoids were in the flowers and umbels (47.9\%-64.2\%). Moreover, HS-SPME allowed the occurrence of isochavicol (29.6 - 34.7\%) as main component in D. crinitus leaves, but it was not detected in the oils, probably because of its solubility in water.

Conclusions: This study demonstrates that HD and HS-SPME modes could be complimentary extraction techniques in order to obtain the complete characterization of plant volatiles.

\section{Background}

Daucus is a genus belonging to the Apiaceae family and consists of about 600 species that are widely distributed around the world. D. carota (carrot) is the main species of the Daucus genus, and its cultivated form, Daucus carota ssp. sativa, is one of the most popular root vegetable crops in the world. Carrots have been reported to be endowed with medicinal properties, i.e., hypotensive, diuretic, carminative, stomachic, and antilipemic

\footnotetext{
* Correspondence: muselli@univ-corse.fr

2Université de Corse, UMR CNRS 6134, Laboratoire Chimie des Produits

Naturels, Campus Grimaldi, BP 52, 20250 Corte, France

Full list of author information is available at the end of the article
}

properties [1-4]. In Algeria, the Daucus genus is represented by species living in dry and uncultivated areas and, among these, D. crinitus Desf. syn. and D. meifolius Brot. are widespread along the Algerian west coast from Tlemcen to Mascara [5]. D. crinitus is characterized by the presence of many subspecies that colonize the sands and cliffs [5]. A survey conducted by herbalists identified that, in folk medicine, a drink made from the roots of D. crinitus is used in decoction to expel the placenta after childbirth, and as a tonic.

Although the phytochemistry of the Daucus genus has been extensively studied (e.g., flavonoids, carotenoids, polyacetylenes, anthocyanins, and volatile constituents), 
only a single study has dealt with the chemical composition of Algerian D. crinitus oil [6]. The oil obtained from the aerial parts is dominated by phenylpropanoid compounds (45.5\%), followed by aliphatic compounds $(17.1 \%)$, and hydrocarbons sesquiterpenes (16.6\%). The main components are isochavicol isobutyrate (39.0\%), an uncommon phenylpropanoid associated with octyl acetate $(12.3 \%)$, and $\beta$-caryophyllene (5.4\%). Moreover, antibacterial and antifungal activities of separated phenylpropanoid esters of the entire oil have been reported.

The essential oils of aromatic herbs are traditionally obtained using hydrodistillation. Because the emitted volatile fraction plays a fundamental role in a plant's life, various novel techniques have been developed for its extraction from plants. Among these, headspace solid phase microextraction (HS-SPME) allows for the rapid fingerprinting of a plant's headspace [7-10], and HS sampling requires the optimization of the extraction parameters to be carried out. As has been previously reported in the literature $[9,10]$, the most effective fibers from vegetable matrices used are those consisting of three polymers: a liquid (PDMS) for the less polar components, and two solids, DVB and CAR, for the more polar components. Several conditions regarding the time and temperature for equilibrium and extraction have been reported, according the plant material analyzed [7-10].

To obtain a better understanding of the volatiles of $D$. crinitus, we investigated the chemical composition of Algerian D. crinitus essential oils extracted using hydrodistillation (HD) from separated organs (i.e., the roots, stems, leaves, flowers, and umbels), and the volatile fractions extracted using HS-SPME from the same plant material. In both cases, the analysis was carried out using gas chromatography (GC) and gas chromatography-mass spectrometry (GC-MS).

\section{Results and Discussion}

\section{Composition of the essential oils}

An analysis of the essential oils from the roots, stems, leaves, flowers, and umbels of D. crinitus harvested in four locations (A-D) identified 72 components, which accounted for $90.9 \%-98.3 \%$ of the total number. Their retention indices and relative percentages are shown in Table 1. Among these, 22 nonterpenic compounds, 12 monoterpenes, nine sesquiterpenes, and four diterpenes were identified. Identification of 33 components was performed by comparing their EI-MS and retention indices with those from the laboratory-produced "Arômes" library; 13 components were identified by comparing their EI-MS and apolar retention indices with those reported in commercial or literature libraries.
However, the main component of the oils in the aerial parts of D. crinitus (53, 84.1\%-40.1\%) remained unidentified, and its identification was carried out using joint information obtained using data from EI-MS and ${ }^{13} \mathrm{C}$ NMR spectroscopy. The EI-MS data of $\mathbf{5 3}$ were close to those of isochavicol 2-methylbutyrate (4-(prop-(1E)enyl)phenyl 2-methylbutyrate) 60, except for the molecular ion $(\mathrm{m} / \mathrm{z}=204 \mathrm{vs} . \mathrm{m} / \mathrm{z}=218$, respectively), and for a signal at $\mathrm{m} / \mathrm{z}=43$, replacing a peak occurring at $\mathrm{m} / \mathrm{z}=57$. These signals suggest the occurrence of an isochavicol-derivative compound that had lost a 14 uma fragment from the acyl part. The ${ }^{13} \mathrm{C}$-NMR spectra acquired from the stem oil from a sample from Bensekrane $(53,84.1 \%)$ exhibited 10 signals, of which, three had double the intensity, assigned from the DEPT spectra of three quaternary carbon atoms (175.60, 149.65, and $135.59 \mathrm{ppm})$, and two aromatic methine carbon atoms (121.46 and $126.65 \mathrm{ppm}$ ), two unsaturated methine carbon atoms (130.15 and $125.78 \mathrm{ppm})$ and two methyl carbon atoms (18.43 and $18.93 \mathrm{ppm})$. These signals confirm the presence of phenylpropanoid compounds, and the formula of $\mathrm{C}_{13} \mathrm{H}_{16} \mathrm{O}_{2}$ was deduced from the DEPT spectra. The identification of isochavicol isobutyrate was unambiguously established from a comparison of its ${ }^{13} \mathrm{C}$-NMR spectral data with those reported in the literature $[6,11]$ and from the identification of isochavicol as a corresponding alcohol from the LAH reduction of the Bensekrane stem oil sample.

Concerning the plant chemistry, two types of essential oils were produced by $D$. crinitus. The root oils were mainly composed of aliphatic compounds (87.0\%-90.1\%), and the aerial part oils (i.e., the leaves, stems, flowers, and umbels) were characterized by the occurrence of phenylpropanoids (43.1\%-88.6\%). The three main aliphatic compounds in the root oils were: dodecyl acetate $\mathbf{5 7}$ (30.3\%-48.2\%), undecane 17 (14.4\%-34.1\%), and dodecanal 35 (16.7\%-26.3\%). It is noticeable that the relative percentage abundance of these compounds differed according to the locality of the sampling; undecane was the major component in the Bensekrane (A) and Sid Abdelli (B) sample oils (34.1\% and 30.9\%, respectively), whereas dodecyl acetate $\mathbf{5 7}$ was identified as the main aliphatic component in the Terni $(\mathrm{C})$ and Beni Snous (D) root oils (48.2\% and $42.3 \%$, respectively).

Conversely, in the oils from the aerial parts, the main components were isochavicol esters. These oils were similar qualitatively, but differed in the relative amounts of their major components. We noted that isochavicol isobutyrate 53 was always the major component (84.1\%$36.5 \%)$, whichever organ was analyzed. The other major components identified were: isochavicol 2-methylbutyrate $\mathbf{6 0}(1.8 \%-17.8 \%)$, pentadecane $\mathbf{5 0}(0.4 \%-15.2 \%)$, zizaene 44 (trace-9.2\%), and undecane 17 (trace-14.2\%). Moreover, except for the stem oil obtained from the 


\begin{tabular}{|c|c|c|c|c|c|c|c|c|c|c|c|c|c|c|c|c|c|c|c|c|c|c|c|c|}
\hline \multirow[t]{2}{*}{ No. ${ }^{a}$} & \multirow[t]{2}{*}{ Components $^{\mathrm{f}}$} & \multirow[t]{2}{*}{$/ \mathrm{RI}_{\mathrm{a}}^{\mathrm{b}}$} & \multirow[t]{2}{*}{$\mathrm{Rl}_{\mathrm{a}}^{\mathrm{c}}$} & \multirow[t]{2}{*}{$\mathrm{RI}_{\mathrm{p}}{ }^{\mathrm{d}}$} & \multicolumn{4}{|c|}{ Roots $^{e}$} & \multicolumn{4}{|c|}{ Leaves $^{\mathrm{e}}$} & \multicolumn{4}{|c|}{ Stems ${ }^{\mathrm{e}}$} & \multicolumn{4}{|c|}{ Umbels $^{\mathrm{e}}$} & \multicolumn{4}{|c|}{ Flowers $^{\mathrm{e}}$} \\
\hline & & & & & A & $B$ & C & D & $A$ & $B$ & $C$ & $\mathrm{D}$ & $A$ & $B$ & $C$ & $\mathrm{D}$ & $A$ & $B$ & $C$ & $\mathrm{D}$ & $A$ & $B$ & $C$ & $\mathrm{D}$ \\
\hline 1 & Nonane & 906 & 902 & 907 & 0.9 & 0.8 & - & - & 0.6 & 0.5 & 0.5 & 0.6 & $\operatorname{tr}$ & $\operatorname{tr}$ & $\operatorname{tr}$ & - & 0.5 & 0.4 & 0.2 & 0.3 & - & - & 0.1 & $\operatorname{tr}$ \\
\hline 2 & $\alpha$-Pinene & 936 & 931 & 1015 & - & - & - & - & 0.5 & 0.4 & 0.1 & - & - & tr & $\operatorname{tr}$ & - & 0.9 & 0.8 & 0.6 & 0.9 & 0.2 & 0.1 & 0.2 & 0.5 \\
\hline 3 & Sabinene & 973 & 966 & 1107 & - & - & - & - & 0.6 & 0.5 & 0.6 & - & - & - & - & - & 0.1 & 0.3 & 0.1 & $\mathrm{tr}$ & 0.3 & 0.4 & 0.6 & 0.2 \\
\hline 4 & $\beta$-Pinene * & 978 & 971 & 1124 & - & - & - & - & 0.1 & 0.1 & 0.1 & - & - & - & - & - & 0.6 & 0.4 & 0.1 & $\mathrm{tr}$ & 0.1 & 0.3 & 1 & 1.5 \\
\hline 5 & Myrcene & 987 & 981 & 1141 & - & - & - & - & 0.6 & 0.3 & 0.5 & - & 0.7 & 0.7 & 0.9 & 0.9 & 0.1 & 0.2 & 0.3 & 0.4 & 0.2 & 0.1 & $\operatorname{tr}$ & $\operatorname{tr}$ \\
\hline 6 & Decane & 993 & 998 & 999 & 0.1 & 0.1 & - & - & $\operatorname{tr}$ & $\operatorname{tr}$ & $\operatorname{tr}$ & $\operatorname{tr}$ & 0.1 & $\operatorname{tr}$ & 0.1 & 0.1 & $\mathrm{tr}$ & 0.1 & $\mathrm{tr}$ & $\mathrm{tr}$ & $\mathrm{tr}$ & 0.1 & 0.1 & $\operatorname{tr}$ \\
\hline 7 & p-Cymene & 1015 & 1012 & 1247 & - & - & - & - & 0.2 & 0.1 & - & - & 0.1 & 0.1 & 6 & 4.2 & 0.7 & 0.4 & 0.3 & $\operatorname{tr}$ & - & - & - & - \\
\hline 8 & 1,8-Cineole * & 1024 & 1020 & 1199 & - & - & - & - & 0.1 & 0.1 & $\operatorname{tr}$ & $\operatorname{tr}$ & tr & - & 0.1 & - & 0.1 & 0.1 & 0.2 & $\mathrm{tr}$ & 0.2 & 0.4 & 0.3 & 0.6 \\
\hline 9 & Limonene & 1025 & 1026 & 1190 & - & - & - & - & 0.9 & 0.6 & 0.3 & - & 0.8 & 0.8 & 0.9 & 1.1 & 1.6 & 1.2 & 1.9 & 2.6 & - & - & - & - \\
\hline 10 & (E)- $\beta$-Ocimene & 1041 & 1037 & 1224 & - & - & - & - & 0.6 & 0.3 & 0.2 & - & 0.1 & $\operatorname{tr}$ & 1.1 & 1.6 & 0.2 & 0.1 & 0.1 & $\operatorname{tr}$ & - & - & - & - \\
\hline 11 & $\boldsymbol{\gamma}$-Terpinene & 1051 & 1049 & 1228 & - & - & - & - & 1.6 & 0.8 & 0.1 & - & 0.1 & 0.1 & 0.2 & 0.5 & 0.3 & 0.2 & 0.2 & $\operatorname{tr}$ & - & - & - & - \\
\hline 12 & 1-Octanol & 1061 & 1056 & 1390 & - & - & - & - & $\operatorname{tr}$ & $\operatorname{tr}$ & & $\operatorname{tr}$ & 0,1 & 0,1 & 0,1 & $\operatorname{tr}$ & 0,2 & 0,2 & 0,1 & 0,1 & $\operatorname{tr}$ & $\operatorname{tr}$ & - & - \\
\hline 13 & 2-Methyl-decane & 1068 & 1065 & 1146 & 0.1 & 0.4 & - & - & $\operatorname{tr}$ & $\operatorname{tr}$ & 0.1 & 0.3 & - & - & - & - & - & - & - & - & - & - & - & - \\
\hline 14 & Nonanal & 1076 & 1074 & 1403 & - & - & - & - & - & 0,2 & $\operatorname{tr}$ & - & $\operatorname{tr}$ & 0,1 & 0,2 & 0,1 & 0,1 & 0,1 & 0,1 & 0,1 & - & - & - & - \\
\hline 15 & $\alpha$-Terpinolene & 1082 & 1079 & 1274 & - & - & - & - & 0.4 & 0.2 & 0.1 & - & 0.3 & 0.3 & 0.5 & 0.4 & 0.3 & 0.1 & 0.2 & 0.1 & 0.1 & 0.2 & 0.4 & 0.3 \\
\hline 16 & Linalool * & 1086 & 1086 & 1528 & - & - & - & - & 0.2 & 0.1 & $\operatorname{tr}$ & - & $\operatorname{tr}$ & $\operatorname{tr}$ & $\operatorname{tr}$ & 0.1 & 0.1 & 0.2 & 0.1 & $\operatorname{tr}$ & $\operatorname{tr}$ & 0.1 & 0.1 & 0.2 \\
\hline 17 & Undecane & 1100 & 1098 & 1101 & 34.1 & 30.9 & 14.4 & 15.3 & 4.1 & 5.1 & 4.5 & 14.2 & $\operatorname{tr}$ & 0.3 & 0.3 & 0.4 & 0.3 & 0.5 & 0.4 & 0.6 & 0.1 & 0.3 & 0.2 & 0.1 \\
\hline 18 & 2-Ethyl hexyl acetate * & 1144 & 1149 & 1420 & - & - & - & - & $\operatorname{tr}$ & $\operatorname{tr}$ & 0.1 & $\operatorname{tr}$ & 0.1 & $\operatorname{tr}$ & 0.2 & 0.1 & - & - & $\operatorname{tr}$ & 0.1 & - & 0.2 & 0.2 & 0.5 \\
\hline 19 & Terpinen 4-ol & 1164 & 1161 & 1586 & - & - & - & - & 0.1 & 0.1 & $\operatorname{tr}$ & - & $\operatorname{tr}$ & 0.1 & 0.3 & 0.4 & 0.3 & 0.3 & 0.1 & $\operatorname{tr}$ & - & - & - & $\operatorname{tr}$ \\
\hline 20 & Octyl acetate * & 1188 & 1187 & 1460 & & & & & 2.3 & 3.0 & 2.8 & 1.0 & 0.2 & 1.0 & 1.9 & 2.5 & 1.0 & 1.2 & 2.2 & 2.4 & & & & \\
\hline 21 & Decanal & 1188 & 1187 & 1483 & 0.3 & 0.2 & - & - & 1.4 & 1.0 & 1.4 & 0.1 & tr & 0.1 & 0.3 & 1 & 0.6 & 0.2 & 1.0 & 1.2 & - & - & - & 0.1 \\
\hline 22 & Dodecane & 1200 & 1198 & 1209 & 0,1 & 0,2 & $\operatorname{tr}$ & 0,1 & $\operatorname{tr}$ & tr & - & - & 0,1 & 0,1 & 0,2 & $\operatorname{tr}$ & - & - & - & - & - & - & - & - \\
\hline 23 & Decanol & 1263 & 1259 & 1729 & - & - & $\operatorname{tr}$ & 0,1 & 0,1 & 0,2 & 0,3 & 0,1 & 0,1 & 0,2 & 0,1 & 0,1 & - & - & - & - & 0,2 & 0,1 & $\operatorname{tr}$ & $\operatorname{tr}$ \\
\hline 24 & Nonanoic acid & 1263 & 1263 & 2119 & - & - & - & - & - & - & - & - & 0,1 & 0,1 & 0,2 & 0,1 & - & - & - & - & 0,1 & 0,1 & 0,1 & 0,1 \\
\hline 25 & Undecanal & 1290 & 1280 & 1610 & $\operatorname{tr}$ & 0,1 & 0,1 & - & $\operatorname{tr}$ & $\operatorname{tr}$ & $\operatorname{tr}$ & 0,1 & 0,2 & 0,1 & 0,1 & $\operatorname{tr}$ & 0,2 & $\operatorname{tr}$ & $\operatorname{tr}$ & $\operatorname{tr}$ & - & - & - & - \\
\hline 26 & Tridecane & 1300 & 1292 & 1305 & 0.7 & 0.5 & 0.9 & 0.4 & 0.1 & $\operatorname{tr}$ & 0.1 & 0.6 & 0.1 & $\operatorname{tr}$ & 0.1 & 0.2 & 0.2 & 0.5 & 0.4 & 0.5 & 0.1 & 0.2 & 0.1 & $\operatorname{tr}$ \\
\hline 27 & n-Octyl isobutyrate & 1329 & 1325 & 1535 & - & - & - & - & $\operatorname{tr}$ & 0,1 & 0,1 & 0,1 & - & - & - & - & 0,2 & 0,1 & 0,2 & 0,2 & 0,1 & 0,1 & 0,1 & 0,1 \\
\hline 28 & Neryl acetate & 1342 & 1336 & 1730 & - & - & - & $\operatorname{tr}$ & - & - & $\operatorname{tr}$ & $\operatorname{tr}$ & 0,1 & 0,2 & 0,4 & 0,2 & 0,1 & 0,1 & 0,1 & 0,1 & 0,1 & $\operatorname{tr}$ & $\operatorname{tr}$ & 0,1 \\
\hline 29 & Decanoic acid & 1347 & 1348 & 2080 & $\operatorname{tr}$ & 0,1 & $\operatorname{tr}$ & $\operatorname{tr}$ & - & - & - & - & - & 0,1 & 0,1 & $\operatorname{tr}$ & 0,1 & 0,1 & $\operatorname{tr}$ & 0,3 & - & - & - & - \\
\hline 30 & Benzyl 2-methyl butyrate & 1360 & 1352 & 1770 & - & 0,2 & - & $\operatorname{tr}$ & $\operatorname{tr}$ & - & - & - & - & - & - & - & - & - & - & - & 0,1 & 0,2 & 0,1 & 0,1 \\
\hline 31 & Geranyl acetate & 1362 & 1359 & 1759 & - & - & - & - & - & - & - & - & - & 0,2 & 0,1 & 0,1 & 0,1 & 0,1 & 0,1 & 0,1 & 0,2 & 0,1 & 0,1 & 0,2 \\
\hline 32 & Undecanol * & 1363 & 1367 & 1816 & - & - & - & - & 0.1 & 0.1 & $\operatorname{tr}$ & 0.1 & 0.1 & 0.1 & 0.3 & 0.4 & 0.4 & 0.2 & 0.1 & $\operatorname{tr}$ & 0.2 & 0.4 & 0.2 & 0.6 \\
\hline 33 & $\alpha$-Copaene & 1379 & 1372 & 1470 & - & - & - & - & $\operatorname{tr}$ & $\operatorname{tr}$ & $\operatorname{tr}$ & $\operatorname{tr}$ & $\operatorname{tr}$ & 0.1 & 0.2 & 0.4 & 0.5 & 0.4 & 0.3 & 0.2 & 0.1 & 0.1 & 0.1 & 0.1 \\
\hline 34 & $\beta$-Bourbonene & 1386 & 1376 & 1520 & - & - & - & - & - & - & - & - & 0,1 & 0,5 & 0,4 & 0,1 & - & - & - & - & - & - & - & - \\
\hline 35 & Dodecanal & 1389 & 1390 & 1695 & 16.7 & 24.6 & 25.1 & 26.3 & 3.1 & 2.1 & 2.1 & 6.7 & 0.5 & 4.0 & 5.0 & 6.1 & 0.1 & $\operatorname{tr}$ & 0.1 & $\operatorname{tr}$ & - & 0.1 & 0.1 & $\operatorname{tr}$ \\
\hline 36 & Tetradecane & 1400 & 1403 & 1408 & - & - & - & - & $\operatorname{tr}$ & 0,1 & 0,1 & 0,1 & 0,1 & 0,1 & 0,2 & 0,1 & $\operatorname{tr}$ & tr & $\mathrm{tr}$ & $\operatorname{tr}$ & - & - & - & - \\
\hline
\end{tabular}


Table 1 Chemical compositions of Daucus crinitus Desf. essential oils from Algeria (Continued)

\begin{tabular}{|c|c|c|c|c|c|c|c|c|c|c|c|c|c|c|c|c|c|c|c|c|c|c|c|c|}
\hline 37 & Longifolene & 1411 & 1409 & 1575 & - & - & - & - & $\operatorname{tr}$ & $\operatorname{tr}$ & 0,1 & $\operatorname{tr}$ & 0,1 & 0,1 & 0,1 & 0,1 & - & - & - & - & 0,1 & 0,1 & 0,1 & 0,1 \\
\hline 38 & $\alpha$-Santalene & 1422 & 1415 & 1569 & - & - & - & - & - & - & - & - & $\operatorname{tr}$ & 0,1 & 0,1 & 0,2 & - & - & - & - & $\operatorname{tr}$ & $\operatorname{tr}$ & $\operatorname{tr}$ & tr \\
\hline 39 & $\beta$-Caryophylene & 1420 & 1424 & 1555 & - & - & - & - & 0.6 & 0.4 & 0.1 & 0.2 & $\operatorname{tr}$ & 0.4 & 0.3 & 0.1 & 5.4 & 3.2 & 3.9 & 5.1 & 0.2 & 0.1 & $\operatorname{tr}$ & 0.1 \\
\hline 40 & Dauca-3,8-diene & 1428 & 1426 & 1680 & - & - & - & - & $\operatorname{tr}$ & $\operatorname{tr}$ & $\operatorname{tr}$ & 0,1 & - & - & - & - & - & - & - & - & 0,2 & $\operatorname{tr}$ & 0,1 & $\operatorname{tr}$ \\
\hline 41 & $\operatorname{trans-\alpha -Bergamotene}$ & 1432 & 1432 & 1575 & - & - & - & - & 0,1 & 0,1 & $\operatorname{tr}$ & $\operatorname{tr}$ & 0,1 & 0,3 & 0,1 & $\operatorname{tr}$ & - & - & - & $\operatorname{tr}$ & 0,2 & $\operatorname{tr}$ & 0,1 & 0,1 \\
\hline 42 & (E) $-\beta$-Farnesene & 1446 & 1443 & 1660 & - & - & - & - & $\operatorname{tr}$ & $\operatorname{tr}$ & $\operatorname{tr}$ & 0,1 & 0,1 & 0,1 & 0,2 & $\operatorname{tr}$ & $\operatorname{tr}$ & $\operatorname{tr}$ & 0,1 & $\operatorname{tr}$ & 0,2 & 0,2 & 0,1 & 0,2 \\
\hline 43 & $\alpha$-Humulene & 1455 & 1451 & 1648 & - & - & - & - & 0.7 & 1.0 & 1.1 & 0.2 & $\operatorname{tr}$ & 0.2 & 0.3 & 0.2 & 0.5 & 0.5 & 0.6 & 0.3 & $\operatorname{tr}$ & 0.1 & $\operatorname{tr}$ & 0.2 \\
\hline 44 & Zizaene * & 1456 & 1463 & 1860 & 1.2 & 1.3 & 2.9 & 2.7 & $\operatorname{tr}$ & $\operatorname{tr}$ & $\operatorname{tr}$ & 1.4 & 1.0 & 1.1 & 1.0 & 1.0 & 5.9 & 5.2 & 6.0 & 9.2 & 6.2 & 8.1 & 9.1 & 8.0 \\
\hline 45 & Dodecanol & 1472 & 1470 & 1754 & 0.2 & 0.2 & - & - & $\operatorname{tr}$ & $\operatorname{tr}$ & 0.1 & $\operatorname{tr}$ & $\operatorname{tr}$ & $\operatorname{tr}$ & 0.1 & $\operatorname{tr}$ & $\operatorname{tr}$ & 0.1 & 0.1 & $\operatorname{tr}$ & 0.2 & 0.1 & 0.1 & $\operatorname{tr}$ \\
\hline 46 & $\alpha$-Curcumene & 1473 & 1474 & 1769 & - & - & - & - & - & - & - & - & 0,1 & 0,1 & 0,2 & 0,2 & 0,1 & 0,2 & 0,2 & 0,1 & 0.2 & $\operatorname{tr}$ & $\operatorname{tr}$ & $\operatorname{tr}$ \\
\hline 47 & Germacrene-D & 1479 & 1478 & 1710 & - & - & - & - & $\operatorname{tr}$ & $\operatorname{tr}$ & 0.1 & 0.1 & 0.1 & 0.5 & tr & $\operatorname{tr}$ & 0.2 & 0.2 & 0.1 & $\operatorname{tr}$ & 0.2 & 0.1 & $\operatorname{tr}$ & 0.2 \\
\hline 48 & $\beta$-Selinene * & 1486 & 1480 & 1685 & - & - & - & - & $\operatorname{tr}$ & $\operatorname{tr}$ & 0.2 & 0.2 & - & 0.1 & $\operatorname{tr}$ & 0.1 & 0.1 & 0.1 & 0.2 & $\operatorname{tr}$ & $\operatorname{tr}$ & 0.1 & $\operatorname{tr}$ & 0.1 \\
\hline 49 & Zingiberene & 1489 & 1483 & 1717 & - & - & - & - & - & - & - & - & $\operatorname{tr}$ & $\operatorname{tr}$ & 0,1 & $\operatorname{tr}$ & - & - & - & $\operatorname{tr}$ & 0,1 & $\operatorname{tr}$ & $\operatorname{tr}$ & 0,1 \\
\hline 50 & Pentadecane & 1500 & 1497 & 1502 & - & - & - & - & 5.1 & 3.5 & 4.1 & 2.2 & 3.0 & 6.0 & 12.6 & 14.5 & 8.5 & 9.5 & 10.5 & 15.2 & 4.1 & 6.2 & 7.3 & 10.1 \\
\hline 51 & $\delta$-Cadinene & 1520 & 1514 & 1736 & - & - & - & - & $\operatorname{tr}$ & $\operatorname{tr}$ & 0.3 & 0.1 & 0.1 & 0.3 & $\operatorname{tr}$ & 0.1 & 0.6 & 0.4 & 0.3 & 0.4 & - & 0.1 & $\operatorname{tr}$ & 0.1 \\
\hline 52 & Geranyl butyrate & 1534 & 1530 & 1877 & - & 0,1 & $\operatorname{tr}$ & 0,1 & - & - & - & - & $\operatorname{tr}$ & 0,1 & 0,2 & 0,2 & - & 0,1 & $\operatorname{tr}$ & 0,1 & 0,2 & $\operatorname{tr}$ & $\operatorname{tr}$ & 0,1 \\
\hline 53 & Isochavicol isobutyrate & 1546 & 1541 & 2134 & - & - & - & - & 44.9 & 35.2 & 35.6 & 25.2 & 84.1 & 57.6 & 41.2 & 36.5 & 51.2 & 50.2 & 50.2 & 40.1 & 65.3 & 55.3 & 58.2 & 62.3 \\
\hline 54 & (Z)-3-Hexenyl benzoate* & 1545 & 1557 & 2059 & 1.9 & 2.3 & 0.1 & 0.1 & 0.1 & 0.2 & 0.1 & 0.1 & 0.1 & 0.4 & 0.2 & 0.1 & 0.5 & 0.6 & 0.5 & 0.6 & 0.1 & 0.2 & 0.3 & $\operatorname{tr}$ \\
\hline 55 & Dodecanoic acid * & 1554 & 1560 & 2474 & 1.9 & 0.3 & - & - & 1.1 & 12.3 & 9.3 & 5.6 & 0.1 & $\operatorname{tr}$ & 0.6 & 1.2 & 0.5 & 0.3 & 0.3 & 0.2 & 0.1 & 0.2 & 0.6 & 0.3 \\
\hline 56 & Caryophyllene oxyde & 1578 & 1572 & 1980 & - & - & - & - & - & - & - & - & $\operatorname{tr}$ & $\operatorname{tr}$ & $\operatorname{tr}$ & $\operatorname{tr}$ & $\operatorname{tr}$ & $\operatorname{tr}$ & 0,1 & 0,1 & - & - & - & - \\
\hline 57 & Dodecyl acetate & 1585 & 1580 & 1882 & 31.7 & 30.3 & 48.2 & 42.3 & 2.5 & 1.5 & 7.2 & 8.9 & 0.3 & 0.2 & 1.9 & 2.3 & $\operatorname{tr}$ & 0.1 & $\operatorname{tr}$ & 0.1 & 1.5 & 1.6 & 1.8 & 1.0 \\
\hline 58 & $\alpha$-Cedrol & 1603 & 1596 & 2115 & - & - & - & - & $\operatorname{tr}$ & $\operatorname{tr}$ & 0.2 & 0.1 & $\operatorname{tr}$ & $\operatorname{tr}$ & 0,1 & $\operatorname{tr}$ & $\operatorname{tr}$ & $\operatorname{tr}$ & $\operatorname{tr}$ & $\operatorname{tr}$ & 0,1 & $\operatorname{tr}$ & $\operatorname{tr}$ & $\operatorname{tr}$ \\
\hline 59 & Hexadecane & 1600 & 1599 & 1598 & - & - & - & - & 0,1 & 0,1 & 0,1 & 0,2 & 0,2 & 0,1 & 0,1 & 0,1 & $\operatorname{tr}$ & $\operatorname{tr}$ & $\operatorname{tr}$ & 0,1 & $\operatorname{tr}$ & 0,1 & 0,1 & $\operatorname{tr}$ \\
\hline 60 & Isochavicol 2-methyl butyrate & 1651 & 1648 & 2255 & 0.2 & 0.3 & 1.5 & 2.3 & 9.7 & 8.9 & 11.3 & 17.8 & 4.2 & 7.8 & 9.1 & 13.2 & 3.2 & 6.5 & 5.5 & 6.1 & 9.8 & 12.5 & 9.6 & 4.8 \\
\hline 61 & $\alpha$-Bisabolol * & 1673 & 1667 & 2202 & - & - & - & - & $\operatorname{tr}$ & 0.1 & 1.6 & 0.2 & $\operatorname{tr}$ & 0.1 & 0.1 & $\operatorname{tr}$ & 0.1 & 0.1 & 0.1 & 0.1 & 0.2 & 0.2 & 0.1 & $\operatorname{tr}$ \\
\hline 62 & Heptadecane & 1700 & 1703 & 1699 & 0.2 & 0.2 & - & - & 3.4 & 2.4 & 6.2 & 2.1 & 0.4 & 6.5 & 2.5 & 2.7 & 5.8 & 4.8 & 3.9 & 4.1 & 2.5 & 1.9 & 2.4 & 1.1 \\
\hline 63 & Benzyl benzoate & 1730 & 1723 & 2121 & 0,1 & 0,1 & 0,2 & $\operatorname{tr}$ & 0,1 & 0,2 & 0,1 & 0,1 & 0,1 & 0,2 & 0,2 & 0,2 & $\operatorname{tr}$ & 0,1 & $\operatorname{tr}$ & $\operatorname{tr}$ & $\operatorname{tr}$ & 0,1 & $\operatorname{tr}$ & tr \\
\hline 64 & Tetradecanoic acid * & 1761 & 1756 & 2649 & 0.3 & 0.2 & 1.1 & 2.0 & 3.1 & 4.4 & 0.2 & 0.8 & 0.1 & 0.3 & 0.7 & 0.4 & 0.1 & 0.5 & 0.5 & 0.2 & 0.3 & 1.0 & 2.3 & 2.0 \\
\hline 65 & Lactarazulene * & 1796 & 1792 & 2430 & 0.1 & 0.4 & 2.7 & 0.4 & 0.7 & 0.5 & 0.1 & $\operatorname{tr}$ & $\operatorname{tr}$ & 0.8 & $\operatorname{tr}$ & 0.1 & - & - & - & - & - & - & 0.1 & $\operatorname{tr}$ \\
\hline 66 & Hexadecanal & 1782 & 1787 & 2108 & 0.3 & 0.2 & 0.2 & 0.3 & 0.1 & 0.6 & $\operatorname{tr}$ & 0.2 & 0.1 & $\operatorname{tr}$ & 0.1 & 0.2 & 0.1 & 0.2 & 0.1 & $\operatorname{tr}$ & - & 0.1 & 0.3 & $\operatorname{tr}$ \\
\hline 67 & Neophytadiene & 1807 & 1807 & 1918 & - & - & - & - & 0.4 & 0.4 & 0.5 & 0.1 & $\operatorname{tr}$ & tr & 0.1 & 0.3 & - & - & - & - & - & 0.4 & 0.8 & 0.4 \\
\hline 68 & Phytone * & 1833 & 1835 & 2131 & - & - & - & - & $\operatorname{tr}$ & 0.7 & 0.3 & $\operatorname{tr}$ & $\operatorname{tr}$ & - & - & - & - & - & - & - & - & - & - & - \\
\hline 69 & Dodecyl pentanoate * & 1843 & 1840 & 2834 & 0.5 & 0.2 & - & - & - & - & & & - & - & - & - & - & - & - & - & - & - & - & - \\
\hline 70 & Isophytol * & 1946 & 1944 & 2316 & & - & - & - & $\operatorname{tr}$ & $\operatorname{tr}$ & 0.6 & 1.1 & $\operatorname{tr}$ & - & - & - & - & - & - & - & $\operatorname{tr}$ & - & - & - \\
\hline 71 & Hexadecanoic acid * & 1951 & 1949 & 2916 & 0.6 & - & - & - & 1.1 & 0.5 & 0.3 & 0.1 & tr & - & - & - & - & - & - & - & 0.2 & - & - & - \\
\hline \multirow[t]{4}{*}{72} & (E)-Phytol * & 2114 & 2102 & 2620 & - & - & - & - & 1.7 & 1.9 & 2.5 & 0.7 & 0.2 & - & - & - & - & - & - & - & 2.3 & - & - & - \\
\hline & Total & & & & 92.2 & 94.2 & 97.4 & 92.4 & 93.2 & 90.9 & 93.8 & 91.9 & 98.3 & 92.5 & 92.4 & 95.1 & 92.7 & 91.4 & 91.1 & 92.3 & 96.9 & 92.3 & 97.5 & 96.6 \\
\hline & Hydrocarbon compounds & & & & 37.5 & 34.8 & 20.9 & 18.9 & 21.4 & 17.4 & 20.2 & 22.8 & 7.5 & 19.7 & 28.8 & 29.7 & 33.4 & 29.7 & 30.9 & 40.1 & 15.4 & 19.2 & 23.0 & 23.5 \\
\hline & Oxygenated compounds & & & & 54.7 & 59.4 & 76.5 & 73.5 & 71.8 & 73.5 & 76.2 & 69.1 & 90.8 & 72.8 & 63.6 & 65.4 & 59.3 & 61.7 & 60.2 & 52.2 & 81.5 & 73.1 & 74.6 & 73.1 \\
\hline
\end{tabular}


Table 1 Chemical compositions of Daucus crinitus Desf. essential oils from Algeria (Continued)

\begin{tabular}{|c|c|c|c|c|c|c|c|c|c|c|c|c|c|c|c|c|c|c|c|c|}
\hline Aliphatic compounds & 88.8 & 89.9 & 90.1 & 87.0 & 28.4 & 37.9 & 39.7 & 44.2 & 6.0 & 19.8 & 28.6 & 33.2 & 19.1 & 19.5 & 18.8 & 26.0 & 10.3 & 13.1 & 16.7 & 19.5 \\
\hline Phenylpropanoid compounds & 2.1 & 2.6 & 1.7 & 2.3 & 54.7 & 44.3 & 47.0 & 43.1 & 88.6 & 65.8 & 50.5 & 49.8 & 54.9 & 57.3 & 56.2 & 46.8 & 75.2 & 68.0 & 68.1 & 64.1 \\
\hline Hydrocarbon non-terpenic compounds & 36,2 & 33.1 & 15.3 & 15.8 & 13.4 & 11.7 & 15.7 & 20.3 & 4.0 & 13.0 & 16.0 & 18.1 & 15.8 & 15.8 & 15.4 & 20.8 & 6.8 & 16.8 & 10.2 & 11.3 \\
\hline Hydrocarbon monoterpenes & - & 1.7 & - & - & 5.5 & 3.3 & 2.0 & - & 2.1 & 2.0 & 9.6 & 8.7 & 4.8 & 3.7 & 3.8 & 4.0 & 0.9 & 1.1 & 2.3 & 2.5 \\
\hline Hydrocarbon sesquiterpenes & 1.3 & - & 5.6 & 3.1 & 2.1 & 2.0 & 2.0 & 2.4 & 1.4 & 4.7 & 3.1 & 2.6 & 12.8 & 10.2 & 11.7 & 15.3 & 7.7 & 9.0 & 9.7 & 9.3 \\
\hline Hydrocarbon diterpenes & - & - & - & - & 0.4 & 0.4 & 0.5 & 0.1 & - & - & 0.1 & 0.3 & - & - & - & - & - & 0.4 & 0.8 & 0.4 \\
\hline Oxygenated non-terpenic compounds & 54,7 & 59.4 & 76.5 & 73.5 & 69.7 & 70.5 & 71.0 & 67.0 & 90.6 & 72.6 & 63.1 & 64.9 & 58.7 & 61.0 & 59.6 & 52.0 & 78.7 & 72.4 & 74.1 & 72.3 \\
\hline Oxygenated monoterpenes & - & - & - & - & 0.4 & 0.3 & - & - & - & 0.1 & 0.4 & 0.5 & 0.5 & 0.6 & 0.4 & - & 0.2 & 0.5 & 0.4 & 0.8 \\
\hline Oxygenated sesquiterpenes & - & - & - & - & - & 0.1 & 1.8 & 0.3 & - & 0.1 & 0.1 & - & 0.1 & 0.1 & 0.2 & 0.2 & 0.3 & 0.2 & 0.1 & - \\
\hline Oxygenated diterpenes & - & - & - & - & 1.7 & 2.6 & 3.4 & 1.8 & 0.2 & - & - & - & - & - & - & - & 2.3 & - & - & - \\
\hline
\end{tabular}

a Order of elution is given on apolar column (Rtx-1). The compounds with an asterisk (*) were only identified in the essential oils.

${ }^{\mathrm{b}}$ Retention indices of literature on the apolar column (IRla) reported from [12-18].

'Retention indices on the apolar Rtx-1 column (Rla).

d Retention indices on the polar Rtx-Wax column (Rlp).

e Percentages are given on the apolar column except for components with identical Rla (percentages are given on the polar column), $\operatorname{tr}=\operatorname{trace}(<0.05 \%)$. Sample locations: Bensekrane (A), Sid Abdeli (B), Terni (C) and Beni Snous (D).

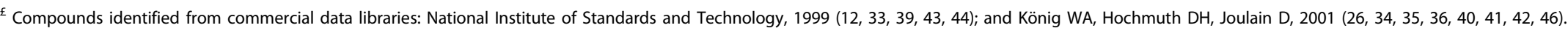


Bensekrane (A) sample, it appeared that the sampling locality had a lesser influence on the variability of the component chemicals of the oils. Finally, it should be noted that the relative percentage abundance of the aliphatic and phenylpropanoid compounds was correlated with the position of the organ on the dressed plant. On moving from the bottom to the top of the plant (i.e., the root, leaf, stem, umbel, and then flower), the relative percentage abundance of aliphatic compounds decreased, while the relative percentage abundance of phenylpropanoids increased. In the same way, we noted that the relative percentage abundance of hydrocarbon sesquiterpenes was higher in the umbels and flower oils (10.2\%-15.3\% and 7.7\%-9.7\%, respectively) than in the other organs (never more than $5.6 \%$ ).

\section{HS-SPME analysis of the volatiles}

The volatiles emitted from the $D$. crinitus roots, leaves, stems, umbels, and flowers harvested in different locations were investigated using HS-SPME under optimized parameters. The optimization of the HS-SPME sampling parameters was carried out using fresh plant material based on the sum of the total peak areas obtained using GC-FID. The maximum sum of the total peak area was acquired for a temperature of $70^{\circ} \mathrm{C}$, an equilibrium time of $60 \mathrm{~min}$, and an extraction time of $30 \mathrm{~min}$ (Table 2). The GC-RI and GC-MS analysis identified 84 components: 45 nonterpenic compounds, 17 monoterpenes, 21 sesquiterpenes, and one diterpene (Tables 3 and 4). Identification of 45 components was performed by comparing their EI-MS and retention indices with those in the laboratory-produced "Arômes" library, and 18 components were identified by comparing their EI-MS data and their apolar retention indices with those reported in commercial or literature libraries.

Regarding the organ contribution to the aromatic plant fingerprint, it should be noted that the volatile constituents were more abundant in the flowers than in the other parts of the plant. Our analysis showed that, for the same organ, the chemical composition of the HS fractions obtained from different localities was qualitatively similar, but differed by the relative percentage abundance of the main components. However, a correlation between the class of compounds and the organ studied was observed: the oxygenated aliphatic compounds were well represented in the roots, hydrocarbon aliphatic compounds were present in the leaves and stems, and phenylpropanoids were present in the flowers and umbels. In particular, the main volatiles from the roots were aliphatic compounds (88.1\%-96.3\%) such as dodecanal 35 (20.6\%-55.4\%), undecane 17 (2.3\%-43.5\%), dodecyl acetate 57 (14.2\%-32.4\%), and dodecanol 45 (1.7\%-8.8\%).

Table 2 Influence of HS-SPME parameters (temperature extraction, equilibrium and extraction times) on the volatiles of Daucus crinitus Desf

\begin{tabular}{|c|c|c|c|c|c|c|c|c|c|c|}
\hline \multirow[b]{3}{*}{ Class of compounds } & \multicolumn{10}{|c|}{ HS-SPME parameters } \\
\hline & & \multicolumn{3}{|c|}{$\begin{array}{c}\text { Equilibrium time } t_{\text {eq }} \min \\
\left(T_{\text {ext }}=70^{\circ} \mathrm{C}-t_{\text {ext }}=15 \mathrm{~min}\right)\end{array}$} & \multicolumn{2}{|c|}{$\begin{array}{c}\text { Extraction time } t_{\text {ext }} \min \\
\left(T_{\text {ext }}=70^{\circ} \mathrm{C}-\mathrm{t}_{\text {eq }}=60 \mathrm{~min}\right)\end{array}$} & \multicolumn{4}{|c|}{$\begin{array}{l}\text { Extraction Temperature } T_{\text {ext }}{ }^{\circ} \mathrm{C} \\
\left(\mathrm{t}_{\text {ext }}=30 \mathrm{~min}-\mathrm{t}_{\text {eq }}=60 \mathrm{~min}\right)\end{array}$} \\
\hline & 20 & 40 & 60 & 80 & 30 & 45 & 30 & 50 & 70 & 90 \\
\hline Hydrocarbon compounds & 37.6 & 58.8 & 53.8 & 49.1 & 69.3 & 52.3 & 85.9 & 76.3 & 69.3 & 62.0 \\
\hline Oxygenated compounds & 56.8 & 37.8 & 44.8 & 47.6 & 30.1 & 38.1 & 11.3 & 22.5 & 30.1 & 36.1 \\
\hline Aliphatic compounds & 74.6 & 69.3 & 78.4 & 72.2 & 80.2 & 67.2 & 74.8 & 79.3 & 80.2 & 76.1 \\
\hline Phenylpropanoid compounds & 2.0 & 3.2 & 1.2 & 2.9 & 0.6 & 2.5 & 0.1 & 0.3 & 0.6 & 0.9 \\
\hline Hydrocarbon non-terpenic compounds & 23.5 & 37.5 & 39.8 & 29.9 & 54.8 & 33.8 & 67.4 & 59.5 & 54.8 & 45.1 \\
\hline Hydrocarbon monoterpenes & 11.9 & 17.2 & 13.1 & 17.6 & 6.7 & 12.1 & 19.3 & 12.6 & 6.7 & 4.1 \\
\hline Hydrocarbon sesquiterpenes & 2.2 & 4.0 & 0.6 & 1.5 & 7.6 & 6.3 & 0.8 & 4.2 & 7.6 & 12.5 \\
\hline Hydrocarbon diterpenes & - & 0.1 & 0.3 & 0.1 & 0.2 & 0.1 & - & - & 0.2 & 0.3 \\
\hline Oxygenated non-terpenic compounds & 52.9 & 35.0 & 39.8 & 45.2 & 26.0 & 35.9 & 9.1 & 20.1 & 26.0 & 31.9 \\
\hline Oxygenated monoterpenes & 3.8 & 2.8 & 4.9 & 2.3 & 1.0 & 2.1 & 2.2 & 2.2 & 1.0 & 0.5 \\
\hline Oxygenated sesquiterpenes & 0.1 & - & 0.1 & 0.1 & 3.1 & 0.1 & - & 0.2 & 3.1 & 3.7 \\
\hline Esters & 4.9 & 5.4 & 5.4 & 7.0 & 9.9 & 4.5 & 3.2 & 8.6 & 9.9 & 12.8 \\
\hline Alcohols & 4.2 & 2.8 & 3.9 & 2.2 & 4.4 & 29.0 & 1.9 & 3.2 & 4.4 & 7.3 \\
\hline Ketones & 0.7 & 0.1 & 0.6 & 0.2 & 0.4 & 0.3 & 0.1 & 0.2 & 0.4 & 0.2 \\
\hline Aldehydes & 43.0 & 25.4 & 28.0 & 35.3 & 10.2 & 25.6 & 3.2 & 8.5 & 10.2 & 11.2 \\
\hline Total identification (\%) & 94.6 & 96.6 & 98.6 & 96.7 & 99.4 & 90.4 & 97.2 & 98.8 & 99.4 & 98.1 \\
\hline Total area $10^{5 \mathrm{a}}$ & 223.2 & 282.8 & 351.0 & 303.1 & 454.1 & 426.4 & 96.8 & 206.4 & 454.1 & 451.2 \\
\hline
\end{tabular}

a Total area is expressed in arbitrary units.

Fresh aerial parts of Daucus crinitus Desf. used for this study were harvested in Bensekrane. 


\begin{tabular}{|c|c|c|c|c|c|c|c|c|c|c|c|c|c|c|c|}
\hline \multirow{2}{*}{$\begin{array}{l}\text { No. } \\
\mathrm{a}\end{array}$} & \multirow[t]{2}{*}{ Components $^{f}$} & \multirow[t]{2}{*}{$/ \mathrm{Rla}^{\mathrm{b}}$} & \multirow[t]{2}{*}{$\mathrm{Rla}^{\mathrm{c}}$} & \multicolumn{4}{|c|}{ Roots $^{d}$} & \multicolumn{4}{|c|}{ Leaves $^{d}$} & \multicolumn{4}{|c|}{ Stems $^{d}$} \\
\hline & & & & A & B & $\mathrm{C}$ & $\mathrm{D}$ & A & B & C & $\mathrm{D}$ & A & B & C & $\mathrm{D}$ \\
\hline $1 a$ & Heptane & 700 & 702 & $0.3 \pm 0.01$ & $\operatorname{tr}$ & - & - & $0.1 \pm 0.01$ & $\operatorname{tr}$ & $\operatorname{tr}$ & $\operatorname{tr}$ & $0.3 \pm 0.07$ & $0.1 \pm 0.01$ & $0.1 \pm 0.01$ & $0.1 \pm 0.01$ \\
\hline $1 b$ & 3-methyl 1-Butanol & 717 & 710 & - & - & - & - & tr & - & - & - & $0.3 \pm 0.01$ & $0.1 \pm 0.01$ & $0.3 \pm 0.01$ & $0.5 \pm 0.07$ \\
\hline $1 c$ & Hexanal & 780 & 767 & $\operatorname{tr}$ & $\operatorname{tr}$ & $\operatorname{tr}$ & - & $\operatorname{tr}$ & $0.1 \pm 0.01$ & $\operatorname{tr}$ & $0.6 \pm 0.07$ & $0.7 \pm 0.07$ & $0.2 \pm 0.01$ & $0.8 \pm 0.07$ & $1.0 \pm 0.14$ \\
\hline $1 d$ & Hex-5-en-1-ol & 820 & 820 & - & - & - & - & $0.3 \pm 0.07$ & $0.5 \pm 0.07$ & $0.2 \pm 0.01$ & $0.9 \pm 0.07$ & $\operatorname{tr}$ & $\operatorname{tr}$ & $0.1 \pm 0.01$ & $0.1 \pm 0.01$ \\
\hline 1e & 2-Heptanone & 871 & 870 & $\operatorname{tr}$ & - & - & - & $\operatorname{tr}$ & $0.3 \pm 0.01$ & & $0.3 \pm 0.01$ & $\operatorname{tr}$ & - & - & - \\
\hline 1 & Nonane & 906 & 902 & $0.3 \pm 0.07$ & $5.5 \pm 0.21$ & - & - & $3.7 \pm 0.14$ & $2.1 \pm 0.14$ & $1.2 \pm 0.14$ & $0.8 \pm 0.14$ & $1.4 \pm 0.14$ & $0.5 \pm 0.07$ & $1.4 \pm 0.21$ & $1.7 \pm 0.28$ \\
\hline 2 & $\alpha$-Pinene & 936 & 931 & - & - & - & - & $0.2 \pm 0.01$ & $2.8 \pm 0.21$ & $0.2 \pm 0.01$ & $0.6 \pm 0.01$ & $6.1 \pm 0.21$ & $1.8 \pm 0.21$ & $6.1 \pm 0.01$ & $2.6 \pm 0.14$ \\
\hline $2 a$ & Camphene & 950 & 943 & - & - & - & - & $0.1 \pm 0.01$ & - & - & - & $0.2 \pm 0.01$ & $0.2 \pm 0.01$ & $0.2 \pm 0.01$ & $0.2 \pm 0.01$ \\
\hline 3 & Sabinene & 973 & 966 & - & $0.1 \pm 0.01$ & - & - & $0.3 \pm 0.01$ & $0.3 \pm 0.01$ & - & $\operatorname{tr}$ & $0.6 \pm 0.07$ & $0.2 \pm 0.01$ & $0.4 \pm 0.01$ & $0.3 \pm 0.01$ \\
\hline $3 a$ & 2-pentyl Furan & 981 & 978 & $0.3 \pm 0.01$ & - & - & - & $\operatorname{tr}$ & - & - & & $0.2 \pm 0.01$ & $\operatorname{tr}$ & $0.2 \pm 0.01$ & $0.1 \pm 0.01$ \\
\hline 5 & Myrcene & 987 & 981 & - & - & - & - & $0.1 \pm 0.01$ & $0.6 \pm 0.14$ & - & $0.2 \pm 0.07$ & $\begin{array}{c}10.6 \pm \\
0.64\end{array}$ & $\begin{array}{c}10.0 \pm \\
0.64\end{array}$ & $7.4 \pm 0.54$ & $7.5 \pm 0.35$ \\
\hline $5 a$ & $\alpha$-Phellandrene & 1002 & 991 & - & - & - & - & $\operatorname{tr}$ & $0.2 \pm 0.01$ & - & - & $0.3 \pm 0.01$ & $0.4 \pm 0.07$ & $0.4 \pm 0.01$ & $0.4 \pm 0.07$ \\
\hline $5 b$ & 3-methyl butyl Isobutyrate & 994 & 997 & - & - & - & - & $0.2 \pm 0.01$ & $\operatorname{tr}$ & $0.1 \pm 0.01$ & - & $\operatorname{tr}$ & $0.1 \pm 0.01$ & $0.1 \pm 0.01$ & $0.1 \pm 0.01$ \\
\hline 6 & Decane & 993 & 998 & $0.2 \pm 0.01$ & $1.2 \pm 0.14$ & $0.1 \pm 0.01$ & - & $\operatorname{tr}$ & $\operatorname{tr}$ & & - & $0.1 \pm 0.01$ & $0.3 \pm 0.01$ & $0.2 \pm 0.01$ & $0.2 \pm 0.01$ \\
\hline $6 a$ & 2-methyl butyl Isobutyrate & 1004 & 999 & - & - & - & - & $0.1 \pm 0.01$ & $\operatorname{tr}$ & $0.2 \pm 0.01$ & - & $0.1 \pm 0.01$ & - & - & $\operatorname{tr}$ \\
\hline 7 & p-Cymene & 1015 & 1012 & - & - & - & - & & $3.5 \pm 0.21$ & - & $0.1 \pm 0.01$ & $2.2 \pm 0.14$ & $0.2 \pm 0.01$ & $2.7 \pm 0.21$ & $3.3 \pm 0.21$ \\
\hline 9 & Limonene & 1025 & 1026 & - & $0.1 \pm 0.01$ & - & - & $0.2 \pm 0.01$ & $2.0 \pm 0.35$ & $0.2 \pm 0.01$ & $0.3 \pm 0.07$ & $7.5 \pm 0.49$ & $\begin{array}{c}11.7 \pm \\
0.64\end{array}$ & $9.9 \pm 0.49$ & $\begin{array}{c}11.1 \pm \\
0.92\end{array}$ \\
\hline 10 & (E)- $\beta$-Ocimene & 1041 & 1037 & - & - & - & - & $\operatorname{tr}$ & $0.7 \pm 0.07$ & $0.1 \pm 0.01$ & $\operatorname{tr}$ & $0.1 \pm 0.01$ & $0.5 \pm 0.01$ & $0.4 \pm 0.01$ & $0.7 \pm 0.01$ \\
\hline 11 & $\gamma$-Terpinene & 1051 & 1049 & - & - & - & - & $0.2 \pm 0.01$ & $7.4 \pm 0.01$ & - & - & $1.6 \pm 0.28$ & $2.7 \pm 0.21$ & $1.6 \pm 0.01$ & $2.3 \pm 0.28$ \\
\hline 12 & 1-Octanol & 1061 & 1056 & - & - & - & - & $0.1 \pm 0.01$ & $\operatorname{tr}$ & - & $\operatorname{tr}$ & $0.2 \pm 0.01$ & $0.1 \pm 0.01$ & $0.1 \pm 0.01$ & $0.2 \pm 0.07$ \\
\hline 13 & 2-methyl Decane & 1068 & 1065 & $0.4 \pm 0.07$ & $6.7 \pm 0.71$ & $0.3 \pm 0.07$ & - & $0.6 \pm 0.07$ & - & - & - & $\operatorname{tr}$ & - & $\operatorname{tr}$ & tr \\
\hline 14 & Nonanal & 1076 & 1074 & - & - & - & - & $\operatorname{tr}$ & $0.4 \pm 0.01$ & - & - & - & $0.3 \pm 0.01$ & $0.5 \pm 0.01$ & $0.8 \pm 0.07$ \\
\hline 15 & $\alpha$-Terpinolene & 1082 & 1079 & - & - & - & - & $0.2 \pm 0.01$ & $9.4 \pm 0.42$ & - & - & $0.2 \pm 0.01$ & $0.3 \pm 0.01$ & $0.3 \pm 0.01$ & $0.5 \pm 0.07$ \\
\hline $15 a$ & 2-Nonanol & 1085 & 1080 & - & - & - & $0.1 \pm 0.01$ & - & - & - & - & $0.1 \pm 0.01$ & $0.4 \pm 0.01$ & $0.4 \pm 0.01$ & $0.5 \pm 0.01$ \\
\hline 17 & Undecane & 1100 & 1098 & $\begin{array}{c}43.5 \pm \\
0.85\end{array}$ & $5.0 \pm 0.35$ & $\begin{array}{c}26.2 \pm \\
0.92\end{array}$ & $2.3 \pm 0.21$ & $\begin{array}{c}11.1 \pm \\
0.57\end{array}$ & $2.9 \pm 0.35$ & $1.2 \pm 0.07$ & $8.9 \pm 0.28$ & $2.7 \pm 0.78$ & $2.8 \pm 0.14$ & $2.1 \pm 0.35$ & $2.6 \pm 0.14$ \\
\hline $17 a$ & Limonene 1,2-epoxide & 1117 & 1113 & - & - & - & - & $\operatorname{tr}$ & $0.1 \pm 0.01$ & - & - & - & - & - & - \\
\hline $17 \mathrm{~b}$ & Camphor & 1123 & 1120 & - & - & - & - & $\operatorname{tr}$ & $0.5 \pm 0.07$ & $0.3 \pm 0.01$ & $0.5 \pm 0.07$ & $0.1 \pm 0.01$ & $0.2 \pm 0.01$ & $0.2 \pm 0.01$ & $0.2 \pm 0.01$ \\
\hline $17 c$ & n-hexyl Isobutyrate & 1127 & 1126 & - & - & - & - & $0.1 \pm 0.01$ & $0.1 \pm 0.01$ & - & $0.1 \pm 0.01$ & - & $0.2 \pm 0.01$ & $0.1 \pm 0.01$ & $\operatorname{tr}$ \\
\hline $17 d$ & Cryptone & 1160 & 1158 & - & - & - & - & $\operatorname{tr}$ & $0.1 \pm 0.01$ & - & - & $0.4 \pm 0.07$ & $0.3 \pm 0.01$ & $0.3 \pm 0.01$ & $0.3 \pm 0.01$ \\
\hline 19 & Terpinen-4-ol & 1164 & 1161 & - & - & - & - & - & - & - & - & $0.3 \pm 0.01$ & $0.6 \pm 0.07$ & $0.3 \pm 0.01$ & $0.4 \pm 0.01$ \\
\hline $19 a$ & 3-methyl Undecane & 1170 & 1165 & $0.1 \pm 0.01$ & $0.5 \pm 0.07$ & $\operatorname{tr}$ & - & - & - & - & - & - & - & - & - \\
\hline $19 b$ & Estragole & 1175 & 1169 & - & - & - & - & $0.2 \pm 0.01$ & - & - & - & $\operatorname{tr}$ & $0.9 \pm 0.01$ & $0.8 \pm 0.14$ & $1.0 \pm 0.14$ \\
\hline 21 & Decanal & 1188 & 1187 & - & $0.1 \pm 0.01$ & - & - & $1.5 \pm 0.02$ & $\begin{array}{c}14.8 \pm \\
0.01\end{array}$ & $7.8 \pm 0.57$ & $2.5 \pm 0.14$ & $1.1 \pm 0.01$ & $1.8 \pm 0.21$ & $2.1 \pm 0.14$ & $2.5 \pm 0.01$ \\
\hline
\end{tabular}


Table 3 Volatile components extracted by HS-SPME from roots, leaves and stems of Daucus crinitus Desf (Continued)

\begin{tabular}{|c|c|c|c|c|c|c|c|c|c|c|c|c|c|c|c|}
\hline 22 & Dodecane & 1200 & 1198 & $0.3 \pm 0.07$ & $0.3 \pm 0.01$ & $\operatorname{tr}$ & - & $0.1 \pm 0.01$ & tr & - & - & $\operatorname{tr}$ & $0.3 \pm 0.01$ & $0.2 \pm 0.01$ & $0.2 \pm 0.01$ \\
\hline $22 a$ & $\gamma$-Octanolide & 1208 & 1208 & - & - & - & - & $0.1 \pm 0.01$ & $0.1 \pm 0.01$ & $0.5 \pm 0.01$ & $0.1 \pm 0.01$ & $0.2 \pm 0.01$ & $0.4 \pm 0.01$ & $0.3 \pm 0.01$ & $0.4 \pm 0.07$ \\
\hline $22 \mathrm{~b}$ & Chavicol & 1219 & 1214 & - & $0.1 \pm 0.01$ & - & - & $0.1 \pm 0.01$ & $0.1 \pm 0.01$ & $0.1 \pm 0.01$ & - & $0.1 \pm 0.01$ & $0.1 \pm 0.01$ & $0.2 \pm 0.01$ & $0.3 \pm 0.01$ \\
\hline $22 c$ & 2-Nonanyl acetate & 1224 & 1220 & - & $0.7 \pm 0.01$ & - & - & - & - & - & - & $\operatorname{tr}$ & $0.2 \pm 0.01$ & $\operatorname{tr}$ & $\operatorname{tr}$ \\
\hline 23 & Decanol & 1263 & 1259 & - & $0.1 \pm 0.01$ & - & $0.4 \pm 0.07$ & $0.2 \pm 0.01$ & $0.8 \pm 0.01$ & $1.8 \pm 0.21$ & - & $1.8 \pm 0.14$ & $2.2 \pm 0.14$ & $1.4 \pm 0.14$ & $1.5 \pm 0.21$ \\
\hline $23 a$ & trans-Anethol & 1262 & 1260 & - & - & - & - & $\operatorname{tr}$ & - & - & - & $\operatorname{tr}$ & $0.1 \pm 0.01$ & $\operatorname{tr}$ & $\operatorname{tr}$ \\
\hline 24 & Nonanoic acid & 1263 & 1263 & - & $0.1 \pm 0.01$ & - & - & - & - & - & - & $0.3 \pm 0.07$ & $0.4 \pm 0.07$ & $0.4 \pm 0.01$ & $0.4 \pm 0.01$ \\
\hline $24 a$ & Benzyl isobutyrate & 1269 & 1266 & - & - & - & - & - & - & - & - & - & - & - & - \\
\hline $24 b$ & Undecan-2-one & 1273 & 1268 & $0.1 \pm 0.01$ & $\operatorname{tr}$ & - & $1.1 \pm 0.14$ & $0.1 \pm 0.01$ & - & - & - & $0.2 \pm 0.01$ & $0.6 \pm 0.07$ & $0.5 \pm 0.01$ & $0.5 \pm 0.01$ \\
\hline 25 & Undecanal & 1290 & 1280 & - & $0.7 \pm 0.21$ & $0.2 \pm 0.01$ & - & - & $\operatorname{tr}$ & $0.2 \pm 0.01$ & - & $\operatorname{tr}$ & $0.3 \pm 0.01$ & $0.2 \pm 0.01$ & $0.3 \pm 0.01$ \\
\hline 26 & Tridecane & 1300 & 1292 & $3.7 \pm 0.21$ & $7.7 \pm 0.85$ & $4.0 \pm 0.35$ & $0.7 \pm 0.07$ & $0.2 \pm 0.01$ & $0.1 \pm 0.01$ & $0.4 \pm 0.01$ & $0.3 \pm 0.07$ & $0.2 \pm 0.01$ & $0.4 \pm 0.01$ & $0.3 \pm 0.01$ & $0.4 \pm 0.01$ \\
\hline $26 a$ & Isochavicol & 1313 & 1315 & - & - & - & - & $0.1 \pm 0.01$ & $0.1 \pm 0.01$ & $0.7 \pm 0.01$ & - & $0.4 \pm 0.07$ & $0.1 \pm 0.01$ & $0.1 \pm 0.01$ & $\operatorname{tr}$ \\
\hline 27 & n-Octyl isobutyrate & 1329 & 1325 & - & - & - & - & $0.1 \pm 0.01$ & $0.2 \pm 0.01$ & $0.1 \pm 0.01$ & - & - & - & - & - \\
\hline 28 & Neryl acetate & 1342 & 1336 & - & - & - & $0.6 \pm 0.01$ & - & - & $0.1 \pm 0.01$ & - & $0.2 \pm 0.01$ & $1.1 \pm 0.21$ & $1.1 \pm 0.01$ & $1.0 \pm 0.14$ \\
\hline 29 & Decannoic acid & 1347 & 1348 & $0.2 \pm 0.01$ & $0.4 \pm 0.01$ & $0.4 \pm 0.01$ & - & - & - & $\operatorname{tr}$ & - & $\operatorname{tr}$ & $0.2 \pm 0.01$ & $0.1 \pm 0.01$ & $\operatorname{tr}$ \\
\hline 30 & Benzyl 2-methyl butyrate & 1360 & 1352 & - & $0.6 \pm 0.07$ & - & - & $\operatorname{tr}$ & - & $\operatorname{tr}$ & - & - & - & - & - \\
\hline 31 & Geranyl acetate & 1362 & 1359 & - & - & - & - & - & - & $0.1 \pm 0.01$ & - & $\operatorname{tr}$ & $0.2 \pm 0.01$ & $0.2 \pm 0.01$ & $0.1 \pm 0.01$ \\
\hline 33 & $\alpha$-Copaene & 1379 & 1372 & - & - & - & - & - & - & - & - & $1.6 \pm 0.14$ & $2.1 \pm 0.14$ & $4.1 \pm 0.01$ & $2.6 \pm 0.14$ \\
\hline 34 & $\beta$-Bourbonene & 1386 & 1376 & - & - & - & - & - & - & - & - & $4.0 \pm 0.42$ & $3.6 \pm 0.21$ & $3.7 \pm 0.01$ & $3.2 \pm 0.28$ \\
\hline 35 & Dodecanal & 1389 & 1390 & $29.3 \pm 1.2$ & $\begin{array}{c}20.6 \pm \\
1.84\end{array}$ & $\begin{array}{c}45.1 \pm \\
1.27\end{array}$ & $\begin{array}{c}55.4 \pm \\
0.99\end{array}$ & $0.4 \pm 0.14$ & $1.5 \pm 0.21$ & $4.4 \pm 0.14$ & $2.1 \pm 0.01$ & $2.7 \pm 0.28$ & $1.3 \pm 0.21$ & $1.2 \pm 0.01$ & $1.4 \pm 0.07$ \\
\hline 36 & Tetradecane & 1400 & 1403 & - & - & - & - & $0.1 \pm 0.07$ & $0.1 \pm 0.01$ & $0.2 \pm 0.01$ & $0.2 \pm 0.01$ & $0.1 \pm 0.01$ & $0.2 \pm 0.01$ & $0.1 \pm 0.01$ & $0.1 \pm 0.01$ \\
\hline 37 & Longifolene & 1411 & 1409 & - & $0.2 \pm 0.01$ & $\operatorname{tr}$ & - & $\operatorname{tr}$ & $0.2 \pm 0.01$ & - & $2.3 \pm 0.21$ & $0.1 \pm 0.01$ & $0.1 \pm 0.01$ & $0.1 \pm 0.01$ & $0.1 \pm 0.01$ \\
\hline 38 & $\alpha$-Santalene & 1422 & 1415 & - & - & $\operatorname{tr}$ & - & - & - & - & $\operatorname{tr}$ & $0.1 \pm 0.01$ & $\operatorname{tr}$ & $\operatorname{tr}$ & $\operatorname{tr}$ \\
\hline 39 & $\beta$-Caryophyllene & 1421 & 1424 & - & - & - & - & $0.8 \pm 0.14$ & $0.1 \pm 0.01$ & - & - & $0.7 \pm 0.14$ & $1.2 \pm 0.07$ & $0.4 \pm 0.01$ & $0.8 \pm 0.07$ \\
\hline 40 & Dauca-3,8-diene & 1428 & 1426 & - & - & - & - & $0.1 \pm 0.01$ & - & - & - & - & - & - & - \\
\hline 41 & trans- $\alpha$-bergamotene & 1432 & 1432 & - & - & - & - & $0.7 \pm 0.01$ & $0.2 \pm 0.01$ & & & $0.2 \pm 0.01$ & $0.2 \pm 0.01$ & $0.2 \pm 0.01$ & $0.2 \pm 0.01$ \\
\hline 42 & (E)- $\beta$-Farnesene & 1446 & 1443 & - & - & - & - & $0.1 \pm 0.01$ & $0.1 \pm 0.01$ & & $0.1 \pm 0.01$ & $0.1 \pm 0.01$ & $0.2 \pm 0.01$ & $0.1 \pm 0.01$ & $0.1 \pm 0.01$ \\
\hline 43 & $\alpha$-Humulene & 1455 & 1451 & - & - & - & - & $0.4 \pm 0.01$ & $0.3 \pm 0.01$ & $1.0 \pm 0.01$ & $0.3 \pm 0.01$ & $0.9 \pm 0.01$ & $0.3 \pm 0.01$ & $0.4 \pm 0.07$ & $0.4 \pm 0.01$ \\
\hline 45 & Dodecanol & 1472 & 1470 & $2.7 \pm 0.01$ & $8.8 \pm 0.42$ & $1.7 \pm 0.28$ & $4.4 \pm 0.35$ & $0.2 \pm 0.01$ & - & - & - & $0.5 \pm 0.01$ & $0.8 \pm 0.21$ & $0.9 \pm 0.01$ & $0.5 \pm 0.07$ \\
\hline 46 & $\alpha$-Curcumene & 1473 & 1474 & - & - & - & - & - & - & - & - & $0.8 \pm 0.14$ & $0.6 \pm 0.01$ & $0.9 \pm 0.01$ & $0.4 \pm 0.01$ \\
\hline 47 & Germacrene-D & 1479 & 1478 & $0.2 \pm 0.01$ & - & - & - & - & - & - & - & $0.8 \pm 0.01$ & $2.1 \pm 0.28$ & $3.3 \pm 0.01$ & $2.1 \pm 0.07$ \\
\hline 49 & Zingiberene & 1489 & 1483 & $0.2 \pm 0.01$ & $0.7 \pm 0.14$ & $0.4 \pm 0.07$ & $0.6 \pm 0.01$ & - & - & - & - & $\operatorname{tr}$ & $0.2 \pm 0.01$ & $\operatorname{tr}$ & $0.1 \pm 0.01$ \\
\hline 50 & Pentadecane & 1500 & 1497 & $0.1 \pm 0.01$ & $0.1 \pm 0.01$ & $0.2 \pm 0.01$ & $0.2 \pm 0.01$ & $\begin{array}{c}51.6 \pm \\
1.77\end{array}$ & $\begin{array}{c}12.8 \pm \\
0.49\end{array}$ & $\begin{array}{c}41.2 \pm \\
1.34\end{array}$ & $\begin{array}{c}40.8 \pm \\
0.57\end{array}$ & $\begin{array}{c}11.9 \pm \\
1.06\end{array}$ & $\begin{array}{c}13.1 \pm \\
0.78\end{array}$ & $\begin{array}{c}14.0 \pm \\
0.28\end{array}$ & $\begin{array}{c}16.0 \pm \\
0.42\end{array}$ \\
\hline 51 & $\delta$-Cadinene & 1520 & 1514 & - & - & - & - & $0.1 \pm 0.01$ & $\operatorname{tr}$ & $1.1 \pm 0.01$ & $0.3 \pm 0.01$ & - & - & - & - \\
\hline 52 & Geranyl butyrate & 1534 & 1530 & $\operatorname{tr}$ & $0.1 \pm 0.01$ & - & $0.2 \pm 0.01$ & - & $0.6 \pm 0.01$ & - & - & $0.3 \pm 0.01$ & $2.0 \pm 0.01$ & $1.7 \pm 0.01$ & $1.6 \pm 0.21$ \\
\hline 53 & Isochavicol isobutyrate & 1546 & 1541 & & $0.4 \pm 0.01$ & $0.2 \pm 0.01$ & $0.2 \pm 0.01$ & $0.2 \pm 0.01$ & $\begin{array}{c}19.9 \pm \\
0.85\end{array}$ & $\begin{array}{c}10.4 \pm \\
1.13\end{array}$ & $\begin{array}{c}19.0 \pm \\
0.85\end{array}$ & $0.2 \pm 0.01$ & $0.7 \pm 0.14$ & $0.4 \pm 0.01$ & $0.2 \pm 0.01$ \\
\hline 56 & Caryophyllene oxyde & 1578 & 1572 & - & - & - & - & - & - & - & - & $0.1 \pm 0.01$ & $\operatorname{tr}$ & $\operatorname{tr}$ & $\operatorname{tr}$ \\
\hline
\end{tabular}


Table 3 Volatile components extracted by HS-SPME from roots, leaves and stems of Daucus crinitus Desf (Continued)

\begin{tabular}{|c|c|c|c|c|c|c|c|c|c|c|c|c|c|c|c|}
\hline 57 & Dodecyl acetate & 1585 & 1580 & $\begin{array}{c}14.2 \pm \\
0.49\end{array}$ & $\begin{array}{l}32.4 \pm \\
1.70\end{array}$ & $\begin{array}{l}16.4 \pm \\
1.06\end{array}$ & $\begin{array}{l}21.7 \pm \\
0.85\end{array}$ & $0.2 \pm 0.01$ & $0.3 \pm 0.01$ & $3.6 \pm 0.21$ & $5.0 \pm 0.21$ & $2.3 \pm 0.28$ & $2.0 \pm 0.01$ & $2.3 \pm 0.21$ & $2.6 \pm 0.07$ \\
\hline 58 & $\alpha$-Cedrol & 1603 & 1596 & - & - & - & - & - & $0.1 \pm 0.01$ & - & - & $1.4 \pm 0.21$ & $1.0 \pm 0.01$ & $0.9 \pm 0.01$ & $0.9 \pm 0.07$ \\
\hline 59 & Hexadecane & 1600 & 1599 & - & - & - & - & $0.3 \pm 0.01$ & $0.1 \pm 0.01$ & $0.3 \pm 0.01$ & $0.3 \pm 0.01$ & $0.5 \pm 0.01$ & $0.4 \pm 0.01$ & $0.2 \pm 0.01$ & $0.3 \pm 0.01$ \\
\hline 60 & $\begin{array}{l}\text { Isochavicol 2-methyl } \\
\text { butyrate }\end{array}$ & 1651 & 1648 & - & - & - & $0.4 \pm 0.01$ & $0.1 \pm 0.01$ & $2.7 \pm 0.01$ & $2.9 \pm 0.01$ & $2.1 \pm 0.14$ & $0.1 \pm 0.01$ & $0.4 \pm 0.01$ & $0.3 \pm 0.01$ & $0.1 \pm 0.01$ \\
\hline 60a & Bisabolol oxide B & 1654 & 1650 & - & - & - & - & $0.2 \pm 0.01$ & & $\operatorname{tr}$ & $0.1 \pm 0.01$ & $3.9 \pm 0.01$ & $2.7 \pm 0.01$ & $2.1 \pm 0.01$ & $1.1 \pm 0.14$ \\
\hline 62 & Heptadecane & 1700 & 1703 & $0.1 \pm 0.01$ & $0.2 \pm 0.01$ & $\operatorname{tr}$ & $0.2 \pm 0.01$ & $\begin{array}{c}20.7 \pm \\
1.56\end{array}$ & $4.1 \pm 0.21$ & $\begin{array}{c}11.9 \pm \\
0.35\end{array}$ & $6.4 \pm 0.57$ & $\begin{array}{c}20.7 \pm \\
0.57\end{array}$ & $\begin{array}{l}13.2 \pm \\
0.42\end{array}$ & $9.0 \pm 0.35$ & $\begin{array}{c}11.2 \pm \\
0.49\end{array}$ \\
\hline 63 & Benzyl benzoate & 1730 & 1723 & $0.1 \pm 0.01$ & $0.7 \pm 0.01$ & $\operatorname{tr}$ & $2.7 \pm 0.14$ & - & - & $0.3 \pm 0.01$ & $0.7 \pm 0.01$ & $0.5 \pm 0.07$ & $0.2 \pm 0.01$ & $0.1 \pm 0.01$ & $0.2 \pm 0.01$ \\
\hline 66 & Hexadecanal & 1782 & 1787 & $0.5 \pm 0.01$ & $0.5 \pm 0.01$ & $0.5 \pm 0.07$ & $0.7 \pm 0.01$ & $0.2 \pm 0.01$ & $0.1 \pm 0.01$ & $0.2 \pm 0.01$ & $\operatorname{tr}$ & $0.1 \pm 0.01$ & $\operatorname{tr}$ & $\operatorname{tr}$ & $0.2 \pm 0.01$ \\
\hline \multirow[t]{14}{*}{67} & Neophytadiene & 1807 & 1807 & $\operatorname{tr}$ & $0.8 \pm 0.01$ & $\operatorname{tr}$ & $3.2 \pm 0.21$ & $0.6 \pm 0.01$ & $0.2 \pm 0.01$ & $0.1 \pm 0.01$ & $0.4 \pm 0.01$ & $0.3 \pm 0.01$ & $\operatorname{tr}$ & $0.1 \pm 0.01$ & $0.2 \pm 0.01$ \\
\hline & $\%$ Identification & & & $\begin{array}{c}96.8 \pm \\
0.57\end{array}$ & $\begin{array}{c}95.4 \pm \\
0.21\end{array}$ & $\begin{array}{l}95.7 \pm \\
0.28\end{array}$ & $\begin{array}{l}95.2 \pm \\
0.07\end{array}$ & $\begin{array}{c}97.3 \pm \\
0.21\end{array}$ & $\begin{array}{c}93.6 \pm \\
0.64\end{array}$ & $\begin{array}{l}93.1 \pm \\
0.21\end{array}$ & $\begin{array}{c}96.3 \pm \\
0.28\end{array}$ & $\begin{array}{l}95.7 \pm \\
0.35\end{array}$ & $\begin{array}{l}92.1 \pm \\
0.07\end{array}$ & $\begin{array}{c}90.9 \pm \\
0.28\end{array}$ & $\begin{array}{c}92.9 \pm \\
0.49\end{array}$ \\
\hline & Total area $\left(10^{5}\right)$ & & & 486.4 & 451.3 & 445.5 & 430.2 & 391.8 & 351.9 & 378.3 & 345.6 & 312.5 & 336.1 & 333.8 & 342.8 \\
\hline & Hydrocarbon compounds & & & 49.4 & 28.4 & 31.2 & 7.3 & 92.8 & 65.1 & 66.9 & 65.4 & 82.8 & 72.9 & 73.9 & 76.4 \\
\hline & Oxygenated compounds & & & 47.4 & 67.0 & 64.5 & 87.9 & 4.5 & 28.5 & 26.2 & 30.9 & 12.9 & 19.2 & 17.0 & 16.5 \\
\hline & Aliphatic compounds & & & 96.3 & 91.8 & 95.1 & 88.1 & 90.8 & 42.0 & 75.7 & 69.3 & 49.4 & 46.1 & 42.6 & 49.1 \\
\hline & $\begin{array}{l}\text { Phenylpropanoid } \\
\text { compounds }\end{array}$ & & & 0.1 & 1.8 & 0.2 & 3.3 & 0.7 & 22.8 & 14.4 & 21.8 & 1.3 & 2.5 & 1.9 & 1.8 \\
\hline & Hydrocarbon non-terpenic com & mpoun & & 49,0 & 27,4 & 30.8 & 3.5 & 88.7 & 37.1 & 64.2 & 60.8 & 43.8 & 34.2 & 31.3 & 37.3 \\
\hline & Hydrocarbon monoterpenes & & & - & 0.2 & - & - & 1.3 & 26.9 & 0.5 & 1.2 & 29.4 & 28.0 & 29.4 & 28.9 \\
\hline & Hydrocarbon sesquiterpenes & & & 0.4 & 0.8 & 0.4 & 0.6 & 2.2 & 0.9 & 2.1 & 3.0 & 9.3 & 10.7 & 13.1 & 10.0 \\
\hline & Hydrocarbon diterpenes & & & - & - & - & 3.2 & 0.6 & 0.2 & 0.1 & 0.4 & 0.3 & - & 0.1 & 0.2 \\
\hline & $\begin{array}{l}\text { Oxygenated non-terpenic } \\
\text { compounds }\end{array}$ & & & 47,4 & 66.2 & 64.5 & 87.9 & 4.3 & 27.7 & 25.9 & 30.3 & 6.9 & 14.4 & 13.2 & 13.6 \\
\hline & Oxygenated monoterpenes & & & - & & - & - & - & 0.7 & 0.3 & 0.5 & 0.8 & 1.1 & 0.8 & 0.9 \\
\hline & Oxygenated sesquiterpenes & & & - & 0.8 & - & - & 0.2 & 0.1 & - & 0.1 & 5.2 & 3.7 & 3.0 & 2.0 \\
\hline
\end{tabular}

a Order of elution is given on apolar column (Rtx-1). Numbers correspond to those in table 1. The compounds that the number is followed by a letter were only identified in HS-fractions.

${ }^{b}$ Retention indices of literature on the apolar column (IRla) reported from [12-18].

C Retention indices on the apolar Rtx-1 column (Rla).

d Percentages (means of three analyses) obtained by GC-FID (on RTX-1: apolar column) with peak-area normalization under optimized HS-SPME parameters: temperature: $70^{\circ} \mathrm{C}$; equilibrium time: 60 min; extraction time: 30 min. Sample locations: Bensekrane (A), Sid Abdeli (B), Terni (C) and Beni Snous (D).

e Total area is expressed in arbitrary units.

${ }^{f}$ Compounds identified from commercial data libraries: National Institute of Standards and Technology, 1999 (1b, 12, 15c, 17a, 19d, 20b); and literature data: König WA, Hochmuth DH, Joulain D, 2001 (3a, 13a, 19b, $19 c, 24 a, 32 b, 35,36,36 a, 38 a, 41,42)$ 
Table 4 Volatile components extracted by HS-SPME from umbels and flowers of Daucus crinitus Desf

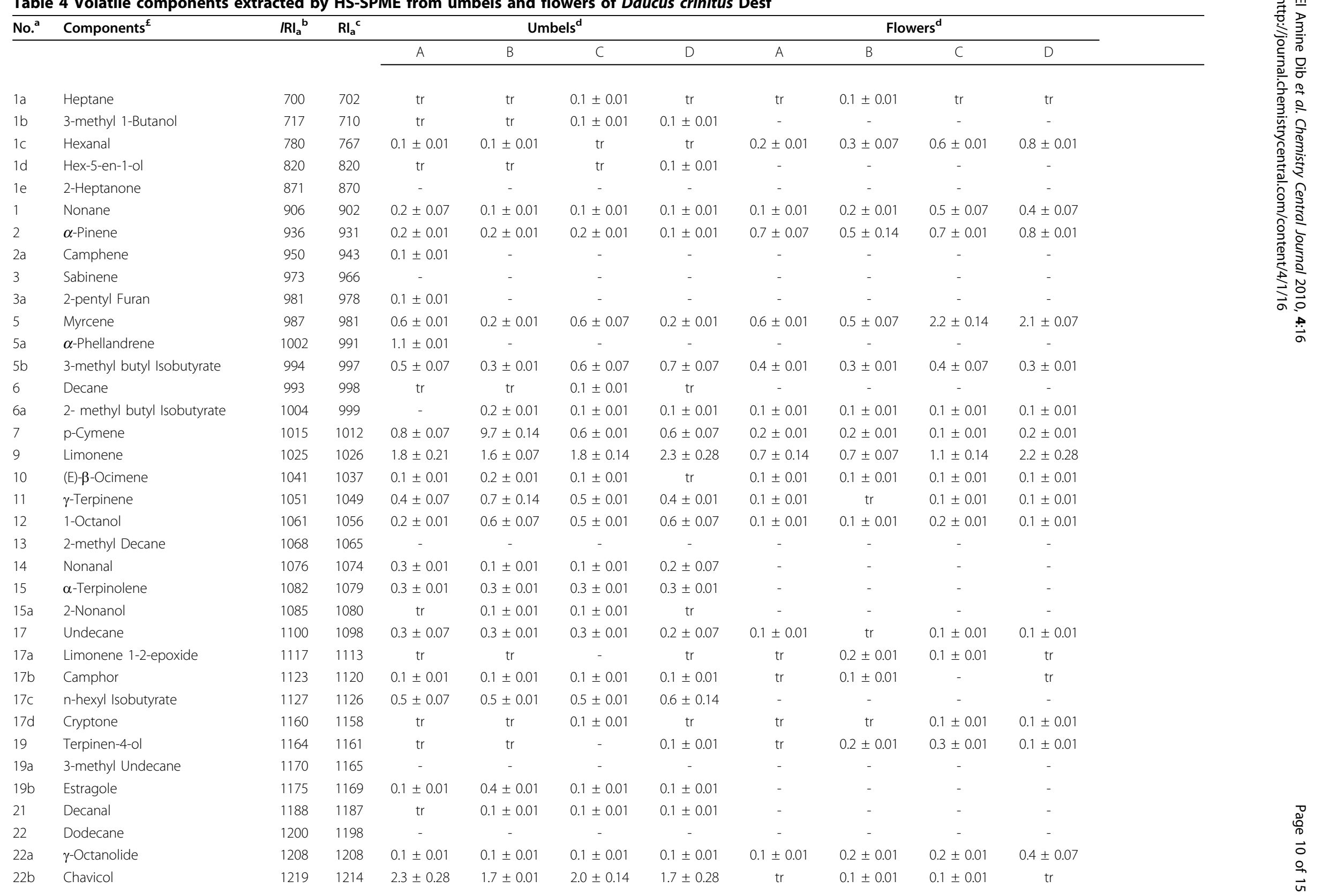


Table 4 Volatile components extracted by HS-SPME from umbels and flowers of Daucus crinitus Desf (Continued)

\begin{tabular}{|c|c|c|c|c|c|c|c|c|c|c|c|c|}
\hline $22 c$ & 2-Nonanyl acetate & 1224 & 1220 & $\operatorname{tr}$ & tr & - & $0.1 \pm 0.01$ & $0.7 \pm 0.14$ & $0.9 \pm 0.01$ & $0.7 \pm 0.01$ & $0.8 \pm 0.14$ & \\
\hline 23 & Decanol & 1263 & 1259 & $0.1 \pm 0.01$ & $0.1 \pm 0.01$ & $0.2 \pm 0.01$ & $0.2 \pm 0.07$ & $0.5 \pm 0.07$ & $0.2 \pm 0.01$ & $0.4 \pm 0.01$ & $0.4 \pm 0.07$ & \\
\hline $23 a$ & Trans-Anethol & 1262 & 1260 & $0.5 \pm 0.01$ & $\operatorname{tr}$ & $0.1 \pm 0.01$ & $0.5 \pm 0.07$ & $0.4 \pm 0.01$ & $0.3 \pm 0.07$ & $0.6 \pm 0.01$ & $0.5 \pm 0.01$ & \\
\hline 24 & Nonanoic acid & 1263 & 1263 & $2.3 \pm 0.21$ & $0.2 \pm 0.07$ & - & - & $0.5 \pm 0.14$ & $0.5 \pm 0.07$ & $0.7 \pm 0.07$ & $0.4 \pm 0.01$ & \\
\hline $24 a$ & Benzyl isobutyrate & 1269 & 1266 & - & - & - & - & $0.3 \pm 0.01$ & $0.5 \pm 0.01$ & $0.5 \pm 0.01$ & $0.5 \pm 0.01$ & \\
\hline $24 b$ & Undecan-2-one & 1273 & 1268 & $0.2 \pm 0.01$ & $0.3 \pm 0.01$ & $0.3 \pm 0.01$ & $0.3 \pm 0.01$ & $0.3 \pm 0.01$ & $0.2 \pm 0.01$ & $0.4 \pm 0.01$ & $0.2 \pm 0.01$ & \\
\hline 25 & Undecanal & 1290 & 1280 & $0.3 \pm 0.01$ & $0.1 \pm 0.01$ & $0.2 \pm 0.01$ & $0.4 \pm 0.01$ & $0.2 \pm 0.01$ & $0.1 \pm 0.01$ & $0.1 \pm 0.01$ & $0.4 \pm 0.01$ & \\
\hline 26 & Tridecane & 1300 & 1292 & $0.1 \pm 0.01$ & $0.1 \pm 0.01$ & $0.2 \pm 0.0$ & $0.1 \pm 0.01$ & $0.2 \pm 0.01$ & $0.1 \pm 0.01$ & $0.2 \pm 0.01$ & $0.2 \pm 0.01$ & \\
\hline $26 a$ & Isochavicol & 1313 & 1315 & $15.2 \pm 1.34$ & $9.1 \pm 0.57$ & $15.5 \pm 0.42$ & $7.1 \pm 0.49$ & $31.8 \pm 1.34$ & $34.7 \pm 1.27$ & $29.6 \pm 0.99$ & $32.9 \pm 1.13$ & \\
\hline 27 & n-Octyl isobutyrate & 1329 & 1325 & $0.4 \pm 0.07$ & $0.2 \pm 0.01$ & $0.3 \pm 0.01$ & $0.2 \pm 0.01$ & $0.8 \pm 0.07$ & $0.7 \pm 0.01$ & $0.4 \pm 0.01$ & $0.5 \pm 0.01$ & \\
\hline 28 & Neryl acetate & 1342 & 1336 & $0.2 \pm 0.01$ & $0.3 \pm 0.01$ & $0.3 \pm 0.01$ & $0.3 \pm 0.01$ & $0.3 \pm 0.01$ & $0.2 \pm 0.01$ & $0.1 \pm 0.01$ & $0.1 \pm 0.01$ & \\
\hline 29 & Decannoic acid & 1347 & 1348 & $0.2 \pm 0.01$ & $\operatorname{tr}$ & $0.1 \pm 0.01$ & $0.1 \pm 0.01$ & - & - & - & - & \\
\hline 30 & Benzyl 2-methyl butyrate & 1360 & 1352 & $\operatorname{tr}$ & $0.2 \pm 0.01$ & $0.1 \pm 0.01$ & $0.1 \pm 0.01$ & $0.3 \pm 0.01$ & $0.1 \pm 0.01$ & $0.5 \pm 0.01$ & $0.3 \pm 0.01$ & \\
\hline 31 & Geranyl acetate & 1362 & 1359 & $0.7 \pm 0.14$ & $0.7 \pm 0.07$ & $0.7 \pm 0.07$ & $0.7 \pm 0.07$ & $0.6 \pm 0.07$ & $0.3 \pm 0.01$ & $0.2 \pm 0.01$ & $0.7 \pm 0.01$ & 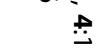 \\
\hline 33 & $\alpha$-Copaene & 1379 & 1372 & $0.3 \pm 0.14$ & $0.4 \pm 0.01$ & $0.5 \pm 0.01$ & $0.4 \pm 0.01$ & $0.6 \pm 0.14$ & $0.4 \pm 0.01$ & $0.6 \pm 0.01$ & $0.6 \pm 0.07$ & \\
\hline 34 & $\beta$-Bourbonene & 1386 & 1376 & $0.1 \pm 0.01$ & $0.1 \pm 0.01$ & $0.1 \pm 0.01$ & $0.1 \pm 0.01$ & $0.1 \pm 0.01$ & $0.2 \pm 0.01$ & $0.2 \pm 0.01$ & $0.1 \pm 0.01$ & \\
\hline 35 & Dodecanal & 1389 & 1390 & $0.4 \pm 0.07$ & $0.6 \pm 0.01$ & $0.6 \pm 0.01$ & $0.5 \pm 0.07$ & $0.5 \pm 0.14$ & $0.5 \pm 0.01$ & $0.8 \pm 0.14$ & $0.4 \pm 0.01$ & \\
\hline 36 & Tetradecane & 1400 & 1403 & $0.1 \pm 0.01$ & $0.1 \pm 0.01$ & $0.1 \pm 0.01$ & $0.1 \pm 0.01$ & $0.1 \pm 0.01$ & $0.1 \pm 0.01$ & $0.1 \pm 0.01$ & $0.1 \pm 0.01$ & \\
\hline 37 & Longifolene & 1411 & 1409 & $\operatorname{tr}$ & tr & tr & $\operatorname{tr}$ & $0.6 \pm 0.01$ & $0.5 \pm 0.01$ & $0.7 \pm 0.01$ & $0.3 \pm 0.07$ & \\
\hline 38 & $\alpha$-Santalene & 1422 & 1415 & - & - & - & - & $0.1 \pm 0.01$ & $0.1 \pm 0.01$ & $0.1 \pm 0.01$ & $0.1 \pm 0.01$ & \\
\hline 39 & $\beta$-Caryophyllene & 1421 & 1424 & $5.1 \pm 0.28$ & $5.8 \pm 0.01$ & $4.2 \pm 0.14$ & $5.4 \pm 0.42$ & $0.1 \pm 0.01$ & $\operatorname{tr}$ & $0.2 \pm 0.01$ & $\operatorname{tr}$ & \\
\hline 40 & Dauca-3,8-diene & 1428 & 1426 & $\mathrm{tr}$ & $\operatorname{tr}$ & $\operatorname{tr}$ & $\mathrm{tr}$ & $0.2 \pm 0.01$ & $0.1 \pm 0.10$ & $0.3 \pm 0.07$ & $0.5 \pm 0.07$ & \\
\hline 41 & Trans- $\alpha$-bergamotene & 1432 & 1432 & $0.1 \pm 0.01$ & $0.1 \pm 0.01$ & $0.1 \pm 0.01$ & $0.2 \pm 0.01$ & $0.7 \pm 0.07$ & $0.4 \pm 0.07$ & $0.6 \pm 0.07$ & $0.5 \pm 0.07$ & \\
\hline 42 & (E)- $\beta$-Farnesene & 1446 & 1443 & $0.4 \pm 0.01$ & $\operatorname{tr}$ & $0.3 \pm 0.01$ & $0.2 \pm 0.01$ & $0.7 \pm 0.07$ & $0.5 \pm 0.07$ & $1.1 \pm 0.01$ & $0.7 \pm 0.01$ & \\
\hline 43 & $\alpha$-Humulene & 1455 & 1451 & $\operatorname{tr}$ & $0.4 \pm 0.01$ & $0.1 \pm 0.01$ & $0.2 \pm 0.07$ & $0.3 \pm 0.01$ & $0.3 \pm 0.01$ & $0.3 \pm 0.01$ & $0.1 \pm 0.01$ & \\
\hline 45 & Dodecanol & 1472 & 1470 & $6.0 \pm 0.14$ & $6.8 \pm 0.49$ & $7.1 \pm 0.35$ & $7.6 \pm 0.42$ & $1.9 \pm 0.28$ & $3.6 \pm 0.14$ & $2.6 \pm 0.07$ & $2.0 \pm 0.14$ & \\
\hline 46 & $\alpha$-Curcumene & 1473 & 1474 & $0.8 \pm 0.21$ & $\operatorname{tr}$ & $0.5 \pm 0.01$ & $0.3 \pm 0.01$ & $0.5 \pm 0.05$ & $0.4 \pm 0.01$ & $0.7 \pm 0.07$ & $0.3 \pm 0.01$ & \\
\hline 47 & Germacrene-D & 1479 & 1478 & $0.2 \pm 0.01$ & $1.0 \pm 0.01$ & $0.9 \pm 0.01$ & $0.7 \pm 0.07$ & $0.5 \pm 0.01$ & $0.2 \pm 0.01$ & $0.5 \pm 0.01$ & $0.5 \pm 0.01$ & \\
\hline 49 & Zingiberene & 1489 & 1483 & $\operatorname{tr}$ & $0.1 \pm 0.01$ & $0.1 \pm 0.01$ & $0.1 \pm 0.01$ & $0.5 \pm 0.01$ & $0.3 \pm 0.01$ & $0.8 \pm 0.07$ & $0.4 \pm 0.01$ & \\
\hline 50 & Pentadecane & 1500 & 1497 & $8.9 \pm 0.57$ & $5.9 \pm 0.20$ & $4.1 \pm 0.28$ & $3.6 \pm 0.21$ & $14.2 \pm 0.49$ & $12.5 \pm 0.35$ & $14.0 \pm 0.64$ & $12.9 \pm 0.49$ & \\
\hline 51 & $\delta$-cadinene & 1520 & 1514 & $0.8 \pm 0.01$ & $0.1 \pm 0.01$ & $0.6 \pm 0.01$ & $0.7 \pm 0.07$ & $0.5 \pm 0.01$ & $0.2 \pm 0.01$ & $0.4 \pm 0.01$ & $0.4 \pm 0.01$ & \\
\hline 52 & Geranyl butyrate & 1534 & 1530 & $\operatorname{tr}$ & $0.4 \pm 0.01$ & $0.5 \pm 0.01$ & $0.4 \pm 0.07$ & $\operatorname{tr}$ & $0.1 \pm 0.01$ & $\operatorname{tr}$ & $0.1 \pm 0.01$ & \\
\hline 53 & Isochavicol isobutyrate & 1546 & 1541 & $26.7 \pm 1.20$ & $49.4 \pm 0.99$ & $40.3 \pm 1.70$ & $51.8 \pm 0.64$ & $14.3 \pm 0.01$ & $15.4 \pm 0.64$ & $18.2 \pm 0.49$ & $19.7 \pm 0.57$ & \\
\hline 56 & Caryophyllene oxyde & 1578 & 1572 & $1.0 \pm 0.01$ & $0.6 \pm 0.01$ & $1.1 \pm 0.01$ & $0.9 \pm 0.01$ & - & - & - & - & \\
\hline 57 & Dodecyl acetate & 1585 & 1580 & $0.7 \pm 0.14$ & $0.6 \pm 0.01$ & $0.6 \pm 0.07$ & $0.7 \pm 0.07$ & $0.4 \pm 0.01$ & $0.5 \pm 0.01$ & $0.3 \pm 0.01$ & $0.2 \pm 0.01$ & \\
\hline 58 & $\alpha$-Cedrol & 1603 & 1596 & $0.6 \pm 0.07$ & $0.3 \pm 0.01$ & $0.4 \pm 0.01$ & $0.6 \pm 0.01$ & $1.9 \pm 0.35$ & $1.4 \pm 0.01$ & $1.1 \pm 0.14$ & $1.4 \pm 0.14$ & ๊ี้ \\
\hline 59 & Hexadecane & 1600 & 1599 & $0.1 \pm 0.01$ & $0.6 \pm 0.01$ & $0.3 \pm 0.07$ & $0.3 \pm 0.01$ & $0.7 \pm 0.01$ & $0.5 \pm 0.01$ & $0.5 \pm 0.01$ & $0.4 \pm 0.01$ & \\
\hline 60 & Isochavicol 2-methyl Butyrate & 1651 & 1648 & $3.1 \pm 0.21$ & $3.6 \pm 0.01$ & $3.4 \pm 0.14$ & $2.3 \pm 0.21$ & $2.3 \pm 0.14$ & $2.3 \pm 0.01$ & $2.0 \pm 0.07$ & $2.6 \pm 0.28$ & \\
\hline $60 a$ & Bisabolol oxide B & 1654 & 1650 & $0.6 \pm 0.01$ & $0.1 \pm 0.01$ & $0.1 \pm 0.01$ & $0.1 \pm 0.01$ & $1.7 \pm 0.01$ & $2.5 \pm 0.01$ & $1.3 \pm 0.01$ & $2.4 \pm 0.21$ & \\
\hline
\end{tabular}


Table 4 Volatile components extracted by HS-SPME from umbels and flowers of Daucus crinitus Desf (Continued)

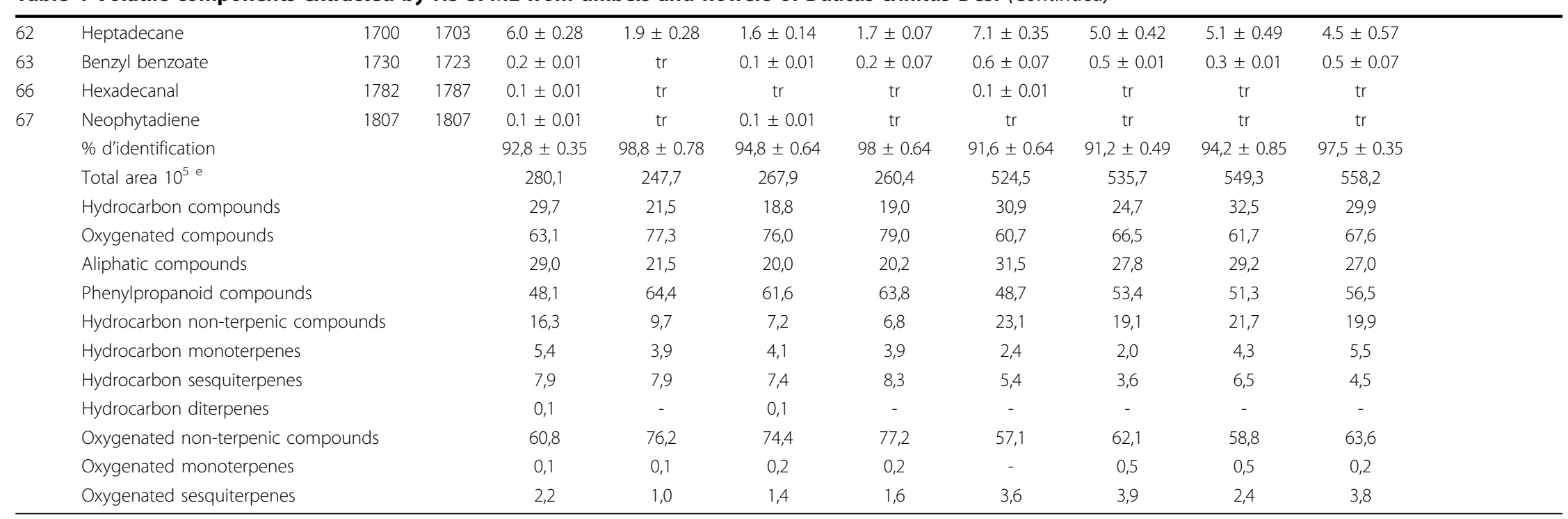

arder of elution is given on apolar column (Rtx-1). Numbers correspond to those in table 1. The compounds that the number is followed by a letter were only identified in HS-fractions.

${ }^{b}$ Retention indices of literature on the apolar column (IRla) reported from [12-18].

' Retention indices on the apolar Rtx-1 column (Rla).

d Percentages (means of three analyses) obtained by GC-FID (on RTX-1: apolar column) with peak-area normalization under optimized HS-SPME parameters: temperature: $70^{\circ} \mathrm{C}$; equilibrium time: 60 min; extraction

time: 30 min. Sample locations: Bensekrane (A), Sid Abdeli (B), Terni (C) and Beni Snous (D).

Total area is expressed in arbitrary units.

${ }^{E}$ The volatile components identified exclusively from the HS-fractions were affected by a letter. Compounds identified from commercial data libraries: National Institute of Standards and Technology, 1999 (1b, 12

15c, 17a, 19d, 20b); and literature data: König WA, Hochmuth DH, Joulain D, 2001 (3a, 13a, 19b, 19c, 24a, 32b, 35, 36, 36a, 38a, 41, 42). 
Regarding the aerial organs, both the leaf and stem volatile fractions were dominated by aliphatic hydrocarbon compounds $(22.2 \%-87.9 \%$ and $27.6 \%-37.9 \%$, respectively) and, in particular, alkanes such as pentadecane 50 (11.9\%-16.0\% and 19.9\%-51.6\%, respectively) and heptadecane 62 (9.0\%-20.7\% and 4.1\%-20.7\%). In addition, hydrocarbon monoterpenes were identified in stems (28.0\%-29.4\%), e.g., limonene 9 (7.5\%-11.7\%) and myrcene 5 (7.4\%-10.6\%), and isochavicol isobutyrate 53 was identified as a volatile emitted from the leaves (0.2\%-19.0\%). The occurrence of phenylpropanoids was established from both the volatile fractions emitted from the umbels and flowers (43.1\%-88.6\%), e.g., isochavicol isobutyrate 53 (26.7\%-51.8\% and $14.3 \%-19.7 \%$, respectively) and isochavicol 26a (7.1\%-15.2\% and $29.6 \%$ $34.7 \%$, respectively). In addition, hydrocarbon aliphatic compounds, in particular pentadecane $\mathbf{5 0}$ and heptadecane 62, were identified and their relative percentage abundance was higher in the volatile fraction extracted from the flowers (the sum was close to 20\%) than from the umbels (the sum was close to $10 \%$ ).

The identification of isochavicol 26a, a compound not present in our MS libraries, was carried out from a comparison of the retention indices and the EI-MS data with those of laboratory-synthesized compounds obtained from the LAH reduction of isochavicol isobutyrate 53, and it was confirmed by a comparison with the ${ }^{13} \mathrm{C}$-NMR data reported in the literature [6]. Isochavicol 26a has been reported to exhibit interesting antiplasmodial activity [6], and to the best of our knowledge, this uncommon phenylpropanoid has not been identified in $D$. crinitus before.

The chemical differences observed between both the essential oils and the volatile fractions extracted using HD and SPME, respectively, can be explained by the fact that the first technique is based on the liquid quasitotal extraction of plant volatiles and the latter technique is controlled by a solid/gas equilibrium step. During hydrodistillation, the most volatile compounds and water-soluble compounds are lost in the gaseous phase and in the hydrolate, respectively, whereas, with HS extraction, it is the fiber affinity of each compound that monitors the sampling of the volatiles. As a consequence, it should be noted that 13 aliphatic compounds (1a-1e, 3a, 5b, 6a, 15a, 17c, 19a, 22c, and 24b) with a low molecular mass and boiling point were identified only in the volatile fractions extracted using HS-SPME. In the same way, isochavicol (26a) was absent in the $D$. crinitus essential oil, but its occurrence in the leaf HS fractions as a main component (7.1\%-34.7\%) can be explained by its solubility in water. Twenty-five compounds (identified by a number followed by a letter in Tables 3 and 4) in total were identified only in the volatile fractions extracted using HS-SPME, and 18 compounds (identified by an asterisk in Table 1) were identified only in the essential oils.

Because the experiments were optimized for the SPME extraction parameters, the extraction temperature was the most important parameter in our plant headspace study. The distribution constants of each component were temperature dependent: the extraction of hydrocarbon monoterpenes and oxygenated sesquiterpenes was improved at a medium temperature $\left(50^{\circ} \mathrm{C}\right)$ and at a high temperature $\left(90^{\circ} \mathrm{C}\right)$, respectively (Table 2 ). The optimal temperature $\left(70^{\circ} \mathrm{C}\right)$ used for the HS extraction was an analytical compromise based on the maximum amount of volatiles extracted. Regarding the comparison of both techniques in terms of the isolation time, HS-SPME was clearly faster (70 $\mathrm{min})$, whereas 300 min was required for hydrodistillation. In the same way, the amount of plant material used for the headspace analysis was less $(1 \mathrm{~g})$, whereas the production of $D$. crinitus oil using hydrodistillation required 200-300 g of plant material. This may be a major reason for the difference in the chemical HS and HD data.

\section{Conclusions}

Several conclusions can be drawn concerning the chemistry of $D$. crinitus from this study.

(i) Two types of essential oil were produced: the root oils, which were mainly composed of aliphatic compounds (87.0\%-90.1\%); and the aerial part oils, which were mainly composed of phenylpropanoids (43.1\%$88.6 \%)$.

(ii) HS-SPME analysis showed a more precise distribution of volatiles in the organs studied: oxygenated aliphatic compounds were well represented in the roots (44.3\%-84.0\%), hydrocarbon aliphatic compounds in the leaves and stems (31.3\%-88.7\%), and phenylpropanoids in the flowers and umbels (47.9\%64.2\%).

(iii) Except for two samples, the location of the samples had a minor influence on the plant volatile production.

Finally, this study has demonstrated that HS-SPME extraction can be considered as an alternative technique for isolating volatiles from aromatic plants.

\section{Experimental}

\section{Plant Material and Oil Isolation}

Samples of each organ (roots, stems, leaves, flowers and umbels) from $D$. crinitus Desf., were collected on November 2008, in Bensekrane (A) $\left[260 \mathrm{~m}, 35^{\circ} 04^{\prime} \mathrm{N} 1^{\circ}\right.$

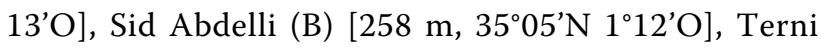
(C) $\left[1199 \mathrm{~m}, 34^{\circ} 47^{\prime} \mathrm{N} 1^{\circ} 21^{\prime} \mathrm{O}\right]$ and Beni Snous (D) [854 $\left.\mathrm{m}, 34^{\circ} 37^{\prime} \mathrm{N} 1^{\circ} 34^{\prime} \mathrm{O}\right]$ forests near Tlemcen, Algeria. 
Voucher specimens were deposited in the herbarium of the Tlemcen University Botanical Laboratory (Voucher number: UBL 05.09). The oils were isolated by hydrodistillation (200 - $300 \mathrm{~g}$ of plant per sample) for $6 \mathrm{~h}$ using a Clevenger-type apparatus [12] according to the European Pharmacopoeia and yielded 0.02 for roots and $0.03-0.04 \%$ for aerial parts.

\section{HS-SPME Conditions}

The single organs of $D$. crinitus were cut roughly with scissors ( $1-2 \mathrm{~cm}$ long) before subjection to HS-SPME. The SPME device (Supelco) coated with divinylbenzene/ carboxen/polydimethylsiloxane (DVB/CAR/PDMS, 30 $\mu \mathrm{m})$ was used for extraction of the plant volatiles. Optimization of conditions was carried out using fresh aerial parts of the plant ( $1 \mathrm{~g}$ in a $20 \mathrm{~mL}$ vial) and based on the number and the sum of total peak areas measured on GC-FID. Temperature, equilibration time and extraction time were selected after nine experiments combining four temperatures $\left(30,50,70\right.$ and $\left.90^{\circ} \mathrm{C}\right)$, four equilibration times (20,40, 60 and $80 \mathrm{~min})$ and three extraction times (15, 30 and $45 \mathrm{~min}$ ). After sampling, SPME fibre was inserted into the GC and GC-MS injection ports for desorption of volatile components ( $5 \mathrm{~min}$ ), both using the splitless injection mode. Before sampling, each fibre was reconditioned for $5 \mathrm{~min}$ in the GC injection port at $260^{\circ} \mathrm{C}$. HS-SPME and subsequent analyses were performed in triplicate. The coefficient of variation $(1.9 \%<$ $\mathrm{CV}<19.8 \%)$ calculated on the basis of total area obtained from the FID-signal for the samples indicated that the HS-SPME method produced reliable results.

\section{Gas Chromatography}

GC analyses were carried out using a Perkin Elmer Autosystem GC apparatus (Walhton, MA, USA) equipped with a single injector and two flame ionization detectors (FID). The apparatus was used for simultaneous sampling to two fused-silica capillary columns $(60$ $\mathrm{m} \times 0.22 \mathrm{~mm}$, film thickness $0.25 \mu \mathrm{m}$ ) with different stationary phases: Rtx-1 (polydimethylsiloxane) and RtxWax (polyethylene glycol). Temperature program: 60 to $230^{\circ} \mathrm{C}$ at $2^{\circ} \mathrm{C} \mathrm{min}^{-1}$ and then held isothermal $230^{\circ} \mathrm{C}(30$ $\mathrm{min})$. Carrier gas: helium $\left(1 \mathrm{~mL} \cdot \mathrm{min}^{-1}\right)$. Injector and detector temperatures were held at $280^{\circ} \mathrm{C}$. Split injection was conducted with a ratio split of 1:80. Injected volume: $0.1 \mu \mathrm{L}$. For HS-SPME-GC analysis, only Rtx- 1 (polydimethylsiloxane) column was used and volatile components were desorbed in a GC injector with a SPME inlet liner (0.75 mm. I.D., Supelco).

\section{Gas Chromatography-Mass Spectrometry}

The oils obtained were investigated using a Perkin Elmer TurboMass Quadrupole Detector, directly coupled to a Perkin Elmer Autosystem XL equipped with two fused-silica capillary columns $(60 \mathrm{~m} \times 0.22$ $\mathrm{mm}$, film thickness $0.25 \mu \mathrm{m}$ ), Rtx-1 (polydimethylsiloxane) and Rtx-Wax (polyethylene glycol). Other GC conditions were the same as described above. Ion source temperature: $150^{\circ} \mathrm{C}$; energy ionization: $70 \mathrm{eV}$; electron ionization mass spectra were acquired with a mass range of 35-350 Da. Oil injected volume: $0.1 \mu \mathrm{L}$. The volatile fractions sampling by HS-SPME were analyzed only on a Rtx-1 capillary column and volatile components were desorbed in a GC injector with a SPME inlet liner (0.75 mm. I.D., Supelco).

\section{Component Identification}

Identification of the components was based (i) on the comparison of their GC retention indices (RI) on non polar and polar columns, determined relative to the retention time of a series of $\mathrm{n}$-alkanes with linear interpolation, with those of authentic compounds or literature data [13-18]; and (ii) on computer matching with commercial mass spectral libraries [15-19] and comparison of spectra with those of our personal library. Relative amounts of individual components were calculated on the basis of their GC peak areas on the two capillary Rtx-1 and Rtx-Wax columns, without FID response factor correction.

\section{NMR analysis}

${ }^{13} \mathrm{C}$-NMR spectra of the stem oil from Bensekrane station (isochavicol isobutyrate 53: 84.1\%) were acquired in deuterated chloroform using a Bruker Avance 400 Fourier Transform spectrometer (Wissembourg, France) operating at $100.13 \mathrm{MHz}$ for ${ }^{13} \mathrm{C}$-NMR and equipped with a $5 \mathrm{~mm}$ probe. All shifts were referred to the internal standard tetramethylsilane (TMS). ${ }^{13} \mathrm{C}$-NMR spectra of the chromatographic fractions were recorded with the following parameters: pulse width, $4 \mu$ s (flip angle, $45^{\circ}$ ); acquisition time, $2.7 \mathrm{~s}$ for $128 \mathrm{~K}$ Data table with a spectral width of $25,000 \mathrm{~Hz}(250 \mathrm{ppm})$; CPD mode decoupling; digital resolution, $0.183 \mathrm{~Hz} / \mathrm{pt}$. The number of accumulated scans was 5000 for a sample (around 40 $\mathrm{mg}$ of the oil in $0.5 \mathrm{~mL}$ of deuterochloroform) depending of the amount of product. Exponential line broadening multiplication $(1 \mathrm{~Hz})$ of the tree induction decay was applied before Fourier Transformation.

\section{Reduction of isochavicol isobutyrate 53}

The isochavicol isobutyrate-rich stem oil $(200 \mathrm{mg})$ from Bensekrane, was dissolved in $\operatorname{dry~}^{2} \mathrm{Et}_{2} \mathrm{O}(4 \mathrm{~mL})$ and was carefully added to a suspension of lithium aluminum hydride (LAH) $(100 \mathrm{mg})$ in dry $\mathrm{Et}_{2} \mathrm{O}(6 \mathrm{~mL})$ at $0^{\circ} \mathrm{C}$. The mixture was stirred at room temperature and then refluxed for $3 \mathrm{~h}$. The reaction mixture was hydrolysed by addition of a solution of $\mathrm{NaOH} 15 \%(2 \mathrm{~mL})$ and cold water. The organic layer was separated, washed 
with water to neutrality, dried over $\mathrm{Na}_{2} \mathrm{SO}_{4}$ and concentrated under vacuum. The mixture $(80 \mathrm{mg})$ exhibited isochavicol (22.3\%) as main components.

\section{Acknowledgements}

The authors are grateful to Prof. M. Bouazza (Botanical Laboratory, Biology Department, Aboubekr Belkaïd University) for the identification of the vegetable matter and Dr. P. Bradesi (University of Corsica, UMR-CNRS 6134, Equipe Chimie et Biomasse) for NMR data acquisition. They are indebted to the Agence Universitaire de la Francophonie (AUF) for providing a research grant to N.D.

\section{Author details}

'Université de Tlemcen, Laboratoire de Chimie Organique, Substances Naturelles et Analyses, BP 119, 13000 Tlemcen, Algérie. Université de Corse UMR CNRS 6134, Laboratoire Chimie des Produits Naturels, Campus Grimaldi, BP 52, 20250 Corte, France.

\section{Authors' contributions}

MAD collected the plant material. MAD, ND, and JMD performed the HD and HS-SPME extractions, obtained the essential oils and the volatile fractions, and participated in the data analysis. MAD, HA, BT, AM, and JC conceived the study and helped draft the manuscript. HA, BT, AM, and JC performed the coordination of the study, and worked on the data analysis and interpretation. All the authors read and approved the final manuscript.

\section{Competing interests}

The authors declare that they have no competing interests.

Received: 30 June 2010 Accepted: 21 September 2010 Published: 21 September 2010

\section{References}

1. Gilani AH, Shaheen E, Saeed SA, Bibi S, Irfanullah SM, Faizi S: Hypotensive action of coumarin glycosides from Daucus carota. Phytomedicine 2000, 7:423-426.

2. Kumarasamy Y, Nahar L, Byres M, Delazar A, Sarker SA: Assessment of biological activities associated with the major constituents of the methanol extract of 'wild carrot' (Daucus carota L.) seeds. J Herbal Pharmacotherapy 2005, 5:61-72.

3. Nicolle C: Lyophilized carrot ingestion lowers lipemia and beneficially affects cholesterol metabolism in cholesterol-fed C57BL/6J mice. Eur J Nutr 2004, 43:237-245.

4. Beloued A: Plantes médicinales d'Algérie. Office des Publications Universitaires, Alger, Algeria 1998

5. Quézel P, Santa S: Nouvelle Flore de l'Algérie et des Régions Désertiques Méridionales 1962-1963. Paris: Éditions du Centre National de la Recherche Scientifique.

6. Lanfranchi DA, Laouer H, El-Kolli M, Prado S, Maulay-Bailly C, Baldovini N: Bioactive Phenylpropanoids from Daucus crinitus Desf. from Algeria. J Agric Food Chem 2010, 58:2174-2179.

7. Bicchi C, Cordero C, Liberto E, Sgorbini B, Rubiolo P: Reliability of fibres in solid-phase microextraction for routine analysis of the headspace of aromatic and medicinal plants. J Chromatogr A 2007, 1152:138-149.

8. Bicchi C, Drigo S, Rubiolo P: Influence of fibre coating in headspace solidphase microextraction-gas chromatographic analysis of aromatic and medicinal plants. J Chromatogr A 2000, 892:469-485.

9. Paolini J, Nasica E, Desjobert JM, Muselli A, Bernardini AF, Costa J: Analysis of Volatile Constituents Isolated by Hydrodistillation and Headspace Solid-phase Microextraction from Adenostyles briquetii Gamisans. Phytochem Anal 2007, 19:266-276.

10. Muselli A, Pau M, Desjobert JM, Foddai M, Usai M, Costa J: Volatile Constituents of Achillea ligustica All. by HS-SPME/GC/GC-MS. Comparison with Essential Oils obtained by Hydrodistillation from Corsica and Sardinia. Chromatographia 2009, 69:575-585.

11. Kubeczka KH, Bartsch A, Ullmann I: Recent studies on essential oils of Apiaceae. Aetherische Oele, Ergeb. Int. Arbeitstag 1982, 158-187.

12. Conseil de l'Europe: Pharmacopée Européenne. France: Maisonneuve S.A. Sainte Ruffine 1996.
13. Jennings W, Shibamoto T: Qualitative analysis of flavour and fragrance volatiles by glass-capillary gas chromatography.Edited by: Jovanovitch HB. Academic Press, New York; 1980.

14. National Institute of Standards and Technology: NIST Chemistry WebBook, NIST Standard Reference Database, 2005, Gaisthersburg, MD. [http://webbook.nist.gov/chemistry].

15. König WA, Hochmuth DH, Joulain D: Terpenoids and Related Constituents of Essential Oils. Library of MassFinder 2.1 University of Hamburg, Institute of Organic Chemistry, Hamburg 2001.

16. National Institute of Standards and Technology. PC version 1.7 of the NIST/EPA/NIH Mass Spectral Library Perkin Elmer Corporation, Norwalk, C 1999.

17. MC Lafferty FW, Stauffer DB: Wiley Registry of Mass Spectral Data. Mass Spectrometry Library Search System Bench-Top/PBM version 3.10d, Palisade, Newfield, 61994.

18. Mc Lafferty FW, Stauffer DB: The Wiley/NBS Registry of Mass Spectral Data. Wiley-Interscience, New York, 41988.

19. Hochmuth D: Mass Spectral Library "Terpenoids and Related Constituents of Essential Oils". Library of MassFinder 3.0 Hamburg, Germany 2006.

20. Adams RP: Identification of essential oil components by gaz chromatography/quadrupole mass spectroscopy. Allured Publishing. Carol Stream, IL, USA 2001

doi:10.1186/1752-153X-4-16

Cite this article as: El Amine Dib et al: Characterization of volatile compounds of Daucus crinitus Desf. Headspace Solid Phase Microextraction as alternative technique to Hydrodistillation. Chemistry Central Journal 2010 4:16

\section{Publish with ChemistryCentral and every scientist can read your work free of charge \\ "Open access provides opportunities to our colleagues in other parts of the globe, by allowing anyone to view the content free of charge." W. Jeffery Hurst, The Hershey Company.}

- available free of charge to the entire scientific community

- peer reviewed and published immediately upon acceptance

- cited in PubMed and archived on PubMed Central

- yours - you keep the copyright

Submit your manuscript here:

http://www.chemistrycentral.com/manuscript/<smiles>c1ccccc1</smiles>

ChemistryCentral 